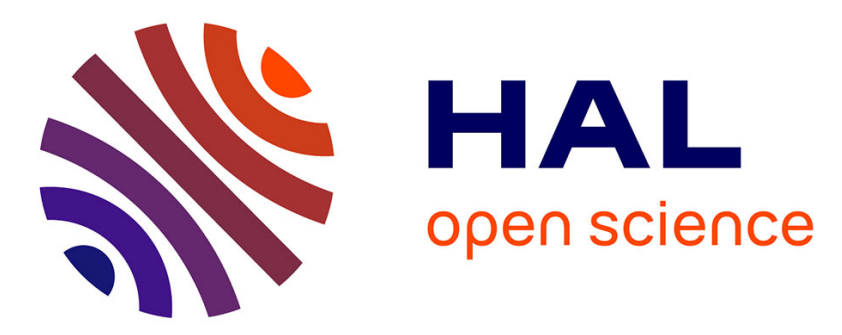

\title{
Temperature Effects on Convection Speed and Steepened Waves of Temporally Developing Supersonic Jets
}

Pierre Pineau, Christophe Bogey

\section{- To cite this version:}

Pierre Pineau, Christophe Bogey. Temperature Effects on Convection Speed and Steepened Waves of Temporally Developing Supersonic Jets. AIAA Journal, 2020, 58 (3), pp.1227-1239. 10.2514/1.J058589 . hal-02504344

\section{HAL Id: hal-02504344 \\ https://hal.science/hal-02504344}

Submitted on 24 Nov 2020

HAL is a multi-disciplinary open access archive for the deposit and dissemination of scientific research documents, whether they are published or not. The documents may come from teaching and research institutions in France or abroad, or from public or private research centers.
L'archive ouverte pluridisciplinaire HAL, est destinée au dépôt et à la diffusion de documents scientifiques de niveau recherche, publiés ou non, émanant des établissements d'enseignement et de recherche français ou étrangers, des laboratoires publics ou privés. 


\title{
Temperature effects on convection speed and steepened waves of temporally-developing supersonic jets
}

\author{
Pierre Pineau * and Christophe Bogey ${ }^{\dagger}$ \\ Univ Lyon, École Centrale de Lyon, INSA Lyon, Université Claude Bernard Lyon I, CNRS, Laboratoire de Mécanique \\ des Fluides et d'Acoustique, UMR 5509, F-69134, Ecully, France
}

\begin{abstract}
Temperature effects on the convection speed of large-scale structures in supersonic temporallydeveloping jets and on the steepened aspect of the acoustic waves generated by these structures are investigated. For that, one isothermal jet at Mach 2 and four others at static temperatures equal to 2 or 4 times that of the ambient medium are simulated at either the same exit velocity or Mach number as the isothermal jet. For all temporally-developing jets, steepened acoustic waves are emitted in the near field, leading to significant values of the pressure skewness and kurtosis factors. Using conditional averages, their formation is directly linked to the supersonic motion of large-scale structures at a convection speed whose ratio with the jet velocity is shown to decrease with temperature. At a higher temperature, this leads to the generation of less skewed acoustic waves for a constant jet velocity. For a constant Mach number, however, this decrease is compensated by the rise of the jet speed, leading to steeper acoustic waves at a higher temperature. Therefore, the modification of the steepened aspect of near-field acoustic waves appears to be related to the change in the convection speed in both cases.
\end{abstract}

\section{Nomenclature}

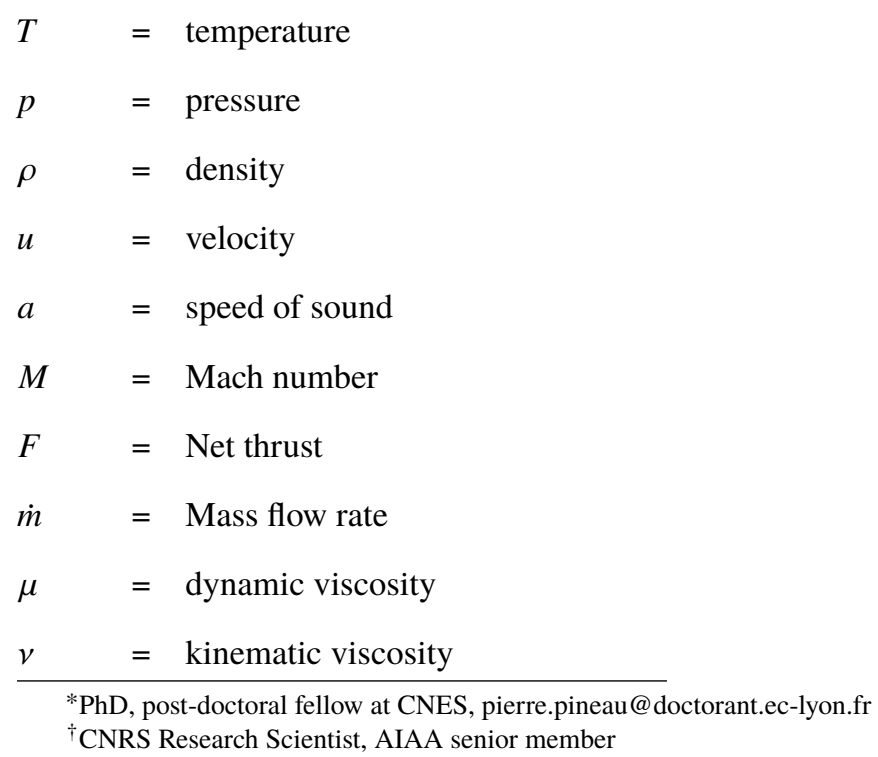




$$
\begin{array}{ll}
r_{s} & =\text { specific ideal gas constant } \\
\gamma & =\text { ratio of specific heats } \\
r & =\text { radial coordinate } \\
\theta & =\text { azimuthal coordinate } \\
z & =\text { axial coordinate } \\
t & =\text { time } \\
\Delta r & =\text { radial mesh spacing } \\
\Delta \theta & =\text { azimuthal resolution } \\
\Delta z & =\text { axial mesh spacing } \\
D & =\text { jet diameter } \\
r_{0} & =\text { jet radius } \\
R_{D} & =\text { diameter-based Reynolds number } \\
\delta_{\theta} & =\text { shear-layer momentum thickness } \\
\dot{\delta}_{\theta} & =\text { momentum thickness growth rate } \\
\dot{\delta}_{\theta, i n c} & =\text { momentum thickness growth rate for an incompressible mixing layer } \\
\delta_{05} & =\text { jet half-width } \\
\alpha & =\text { Mach wave radiation angle }
\end{array}
$$

Subscripts

$$
\begin{array}{ll}
\infty & =\text { ambient quantity } \\
j & =\text { initial value of the jet parameters } \\
c & =\text { potential core closure } \\
\text { axis } & =\text { centerline quantity } \\
\text { cond } & =\text { conditionally averaged } \\
\text { trig } & =\text { conditional averages trigger }
\end{array}
$$
speed $u_{c}$ of coherent structures inside the jet is higher than the ambient sound speed $a_{\infty}$. In this case, straight, elongated 
wavefronts are emitted from the jet and propagate downstream at the angle $\alpha$ given by

$$
\cos \alpha=\frac{a_{\infty}}{u_{c}}
$$

Investigating the effects of temperature on Mach wave radiation is challenging given that the speed of sound $a_{j}$ inside the jet increases with the static temperature $T_{j}$ as $a_{j}=\sqrt{\gamma r_{s} T_{j}}$, where $\gamma$ is the ratio of specific heat and $r_{s}$ is the specific ideal gas constant. In the literature, two different approaches are usually adopted to study temperature effects. A first possibility is to work at a fixed Mach number $M_{j}=u_{j} / a_{j}$, where $u_{j}$ is the jet speed. This approach is natural from an experimental perspective since it amounts to keeping the same nozzle pressure ratio while varying the stagnation temperature. It has been followed in the studies of Seiner et al. [3], Krothapalli et al. [4], and Mora et al. [9], for instance. In that case, the jet velocity increases with temperature, leading to a strengthening of the acoustic waves radiated by the jet. In addition, the peak of sound emission is shifted toward higher angles relative to the jet direction due to the rise of the convection speed. These effects are clearly visible in the results of Seiner et al. [3], who performed measurements in the sound fields of Mach 2 jets. In this study, the acoustic Mach number of the jets, defined as $M_{a}=u_{j} / a_{\infty}$, however varies from 1.3 to 3 , which makes it difficult to isolate the effects of temperature given the strong dependence of jet noise on the exhaust velocity. For that reason, it can be useful to investigate temperature effects for a fixed jet speed, as in the work of Tanna et al. [1, 2] or of Panda [10] for jets at $M_{a} \simeq 1.5$. In that case, the pressure levels radiated at all angles decrease with temperature, revealing a weakening of the sound sources.

The influence of temperature on the formation of steepened, nonlinear acoustic waves near the jets is currently not well understood. When these waves propagate to the far field, they are expected to contribute to the formation of crackle noise [11], which is an unpleasant perception effect. It is known that the pressure levels near highly supersonic jets are sufficiently high that nonlinear propagation effects can occur, leading to a gradual steepening of the wavefronts $[12-14]$. However, steepened waves have been reported in the near vicinity of the jet flow in experimental measurements [9, 15, 16], as well as in numerical simulations [17.-19], that is, too close to be only the result of nonlinear propagation effects. This has led investigators [13, 20] to propose that these waves are produced, at least to a certain extent, by high-intensity events occurring inside the turbulent flow. Unfortunately, there is currently no comprehensive description of the mechanisms involved in their steepening at the source. Since the inclination angles of steepened wavefronts are very close to that of Mach waves, it has been suggested that they constitute a particular, nonlinear case of this phenomenom [18, 19, 21, 22]. Thus, given the influence of temperature on Mach wave radiation, it is reasonable to expect that the mechanisms involved in wave steepening at the source are also affected by temperature.

Temperature effects on crackle noise have been studied by Mora et al. [9], who carried out measurements in the near field of hot and cold supersonic jets at $M_{j}=1.5$. For the hot jet, the skewness and kurtosis factors of the pressure fluctuations and their time-derivative are higher than for the cold jet, indicating that the radiated acoustic waves are 
more strongly nonlinear. These results are in agreement with those of Krothapalli et al. [4], who performed optical visualizations of supersonic jets at $M_{j}=2$ and at stagnation temperatures ranging from $580 \mathrm{~K}$ to $1250 \mathrm{~K}$. At a higher temperature, they observed that more and more steepened waves are present close to the jets. They suggested that these shock-like waves are the consequence of micro explosions caused by the intrusion of cold lumps of ambient fluid entrained in the hot jet core and convected at supersonic speeds. In the two aforementioned studies, the Mach number of the jets is constant, so that the jet speed increases with the temperature. Therefore, it is difficult to conclude whether these results constitute an actual effect of temperature, or if it merely results from the rise of exhaust speed when the jet is heated. In their initial work on jet crackle, Ffowcs Williams et al. [11] reported no clear influence of jet temperature on the formation of steepened acoustic waves for a constant velocity. They however recognized that the range of temperature ratios considered is not sufficiently wide in order to make a definitive conclusion. Since then, very few studies have focused on temperature effects on the formation of steepened waves for a constant jet speed. One exception is the work of Nichols et al. [23], who performed simulations of two hot jets at an acoustic Mach number of 2.7 and at static temperature ratios $T_{j} / T_{\infty}$ of 2.46 and 3.03. In these simulations, the skewness factor of the pressure fluctuations obtained near the hottest jet is very close to that obtained for the other jet. However, given the proximity of the two temperatures, further investigations are needed.

In the present study, the effects of temperature on the steepened waves formed near supersonic jets are therefore investigated by considering the effects of a temperature rise at a constant Mach number, but also at a constant speed. In particular, the importance of the convection speed, which has proven to be a key parameter for the study of crackle [18, 24] is explored. Any parametric investigation of temperature effects in high-speed jets requires a meticulous control over the flow conditions at the nozzle exit. Indeed, the shape of the velocity profile, the turbulence rates, as well as the state of the nozzle boundary layer can affect noise generation [25-28]. In supersonic jets, an additional complexity comes from the formation of shock cells in and downstream of the nozzle. These shock cells can alter the state of the nozzle boundary layer, modify the location of the mixing layers, and cause variations of the convection speed. To prevent their formation, one possibility is to consider jets exhausting at pressure matched conditions from nozzles that are designed using the method of characteristics as in the study of Nonomura et al. [28] for jets at $M_{j}=2$. This approach is however costly when different Mach numbers are considered, since it requires the design and the modelling of one nozzle for each Mach number. Furthermore, these nozzles will generate weak shocks since the method of characteristics does not take into account the presence of boundary layers. Another possibility is to consider temporally-developing axisymmetric mixing layers as simplified models for supersonic shock-free jets, as previously done by the authors to investigate sound generation mechanisms [22, 29]. Indeed, these simulations allow us to vary the jet speed and temperature, hence Mach number, by merely prescribing different initial conditions. This enables us to perform parametric studies at a reasonable cost and with a better control over the initial conditions than in simulations of spatially-developing jets. Temporal simulations are performed by imposing periodicity conditions at the axial boundaries of the computational domain, so 
Table 1 Flow parameters

\begin{tabular}{cccccccccc}
\hline \hline Jet & $T_{j} / T_{\infty}$ & $M_{j}$ & $M_{a}$ & $M_{c}$ & $R e_{D}$ & $\delta_{\theta} / r_{0}$ & $u_{z}^{\prime}(t=0) / u_{j}$ & $t_{f} u_{j} / r_{0}$ & $n_{\text {run }}$ \\
\hline jetT1Mj2 & 1 & 2 & 2 & 1 & 12,500 & 0.018 & 0.02 & 70 & 4 \\
jetT2Mj2 & 2 & 2 & 2.8 & 1.17 & 12,500 & 0.018 & 0.02 & 100 & 3 \\
jetT4Mj2 & 4 & 2 & 4 & 1.33 & 12,500 & 0.018 & 0.02 & 100 & 3 \\
jetT2Ma2 & 2 & 1.4 & 2 & 0.82 & 12,500 & 0.018 & 0.02 & 60 & 3 \\
jetT4Ma2 & 4 & 1 & 2 & 0.67 & 12,500 & 0.018 & 0.02 & 60 & 3 \\
\hline \hline
\end{tabular}

that the flow develops over time from a given initial state. Although these model flows are not jets, strictly speaking, as they do not exhaust from a nozzle nor spread in space, they will be designated as temporal jets in the following in order to facilitate the connection between the present results and those from the literature.

One isothermal and four hot temporally-developing jets are computed at a diameter based Reynolds number of 12,500 by large-eddy simulations. The isothermal jet is at $T_{j}=T_{\infty}$ and has a Mach number $M_{j}=M_{a}=2$. The hot jets have static temperatures of $2 T_{\infty}$ and $4 T_{\infty}$, and either a Mach number $M_{j}=2$ or an acoustic Mach number $M_{a}=2$. This set of simulations allows us to investigate the effects of a temperature rise when the jet speed varies or remains constant. The temperature effects on the steepened aspect of the waves are explored by comparing the pressure levels, skewness and kurtosis factors in the near acoustic field. They are related to changes in the flow by computing, in particular, conditional averages of the generation process of steepened waves. These averages indeed allow us to highlight flow features which are directly related to noise generation, including the structures which produce the waves, as well as their convection speed [19].

The paper is organized as follows. First the different jet parameters are introduced and the numerical procedure used for their computations is described in section [II The simulations results are then presented in section [II] and include snapshots, statistical results such as the pressure spectra, skewness and kurtosis factors, as well as conditional averages. Finally, concluding remarks are given in section IV] and the conditional averaging procedure is applied to a simulation of a spatially-developing jet in appendix in order to verify that the flow structures in temporally-developing flow are similar to those obtained in spatially-developing jets.

\section{Numerical methodology}

\section{A. Jet parameters}

The five temporally-developing jets whose parameters are given in Table 1 are simulated. Their diameter-based Reynolds number $\operatorname{Re}_{D}=u_{j} D / v_{j}$ is equal to 12,500 , where $D=2 r_{0}$ is the initial jet diameter, and $v_{j}=\mu_{j} / \rho_{j}$ is the centerline kinematic viscosity, computed from the Sutherland law. Three of these jets have a Mach number $M_{j}$ of 2 . The static temperatures of these jets, which are labelled jetT1Mj2, jetT2Mj2 and jetT4Mj2, are equal to 1,2 , and 4 times the ambient temperature $T_{\infty}=293 \mathrm{~K}$. The isothermal jet constitutes the reference case of this study, while jetT2Mj2 and 
(a)

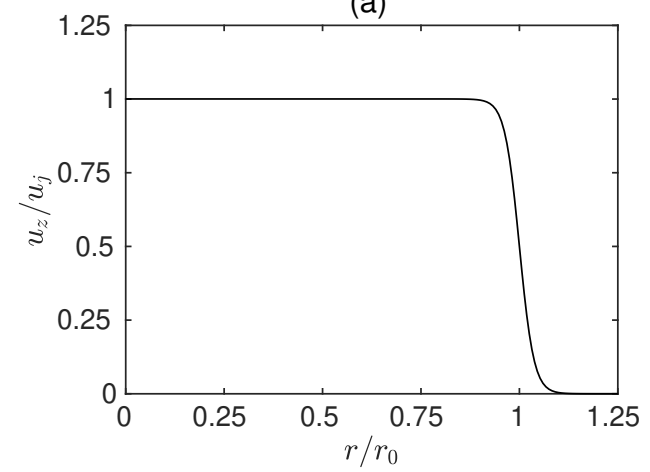

(b)

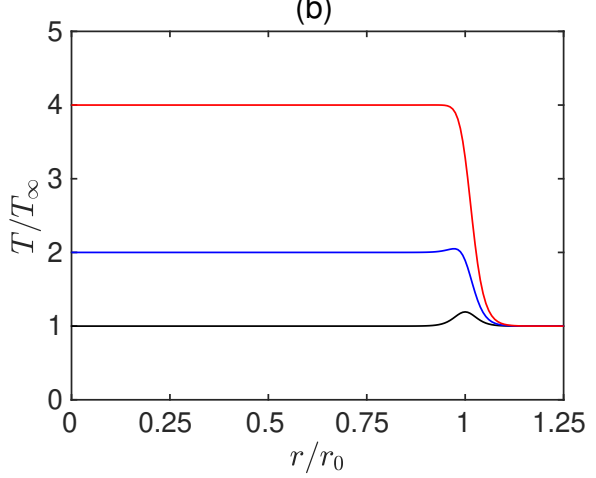

Fig. 1 Initial radial profiles of (a) axial velocity and (b) static temperature for $M_{j}=2$ and $-T_{j} / T_{\infty}=1$, $T_{j} / T_{\infty}=2$, and $-T_{j} / T_{\infty}=4$.

jetT4Mj2 allow us to characterize the effects of a temperature rise at a constant Mach number $M_{j}$. In that case, the jet speed increases with the temperature, leading to acoustic Mach numbers $M_{a}$ of 2.8 and 4 for jetT2Mj2 and jetT4Mj2. In order to isolate the effects of temperature, it is also useful to investigate the effects of a temperature rise at a constant jet speed. This is done here by simulating two hot jets at $T_{j}=2 T_{\infty}$ and $4 T_{\infty}$ at the same speed as the isothermal jet, leading to $M_{a}=2$ in both cases. Since the speed of sound increases with temperature, the Mach numbers of the jets, labelled jetT2Ma2 and jetT4Ma2, are equal to 1.4 and 1 , respectively.

At initial time, the velocity field is initialized with the hyperbolic tangent profile represented in Fig. 1 (a). The shear-layer momentum thickness $\delta_{\theta}$ of this profile has been chosen such that $\delta_{\theta} / r_{0}=2 / \sqrt{\operatorname{Re}_{D}}=0.018$, following an empirical relation obtained for initially-laminar subsonic jets [30]. The ambient pressure $p_{\infty}$ is equal to $10^{5} \mathrm{~Pa}$, and the initial temperature profiles are determined from the Crocco-Busemann relation

$$
\frac{T}{T_{j}}=\frac{T_{\infty}}{T_{j}}-\left(\frac{T_{\infty}}{T_{j}}-1\right) \frac{u_{z}}{u_{j}}+\frac{\gamma-1}{2}\left(\frac{u_{j}}{a_{j}}\right)^{2} \frac{u_{z}}{u_{j}}\left(1-\frac{u_{z}}{u_{j}}\right)
$$

and are plotted in figure 1 (b) for jetT1Mj2, jetT2Mj2 and jetT4Mj2. The initial temperature profiles for jetT2Ma2 and jetT4Ma2 are not provided, as they do not significantly differ from those for the jets at $M_{j}=2$. For jetT2Mj2 and jetT4Mj2, the temperature is equal to $T_{j}$ in the jet core and decreases down to $T_{\infty}$ farther away from the flow. For the isothermal jet, a slight temperature excess is visible around $r=r_{0}$. It is caused by compressibility effects, as derived from relation (2). Finally, low-amplitude velocity perturbations are added at $t=0$ inside the jet shear layers, in order to favor the flow transition from a laminar to a turbulent state. They are vortex rings of random azimuthal mode, phase and amplitude [31]. Their mean amplitude has been arbitrarily tuned so that the initial turbulence rate $u_{z}^{\prime}(t=0) / u_{j}$ is of $2 \%$ for each jets.

A summary of the different effects of increasing temperature on the flow properties is given in Fig. 2, where the initial conditions of the five simulated jets are represented in the velocity-temperature plane, along with isocontours of the Mach number $M_{j}$. This diagram highlights the difficulty to isolate any particular temperature effects. Notably, 


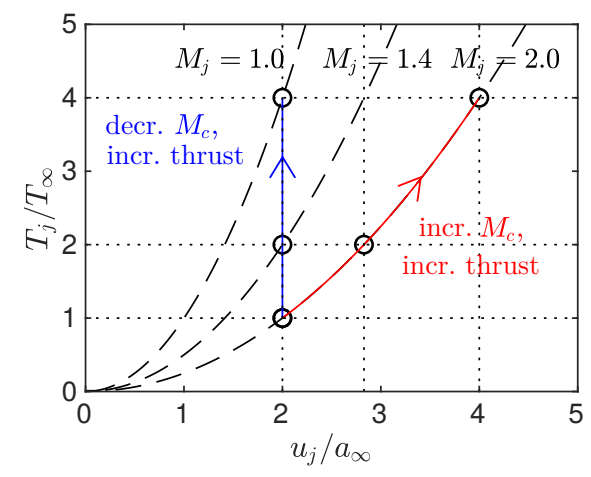

Fig. 2 Initial conditions for the jets at $-M_{a}=2$ and $-M_{j}=2 ;---$ isocontours of Mach number for $M_{j}=1,1.4$ and 2 .

the mass flow rate, defined as $\dot{m}=\pi D^{2} \rho_{j} u_{j} / 4$, is not the same for the five jets, yielding different values of the net thrust $F=\dot{m} u_{j}$ which would be produced by spatially-developing jets at the same exhaust conditions. The latter can be expressed as

$$
F=\frac{\pi}{4} R e_{D}^{2} \frac{r_{s}}{p_{\infty}} T_{j} \mu_{j}^{2}\left(T_{j}\right)
$$

and is only a function of the jet static temperature, since the five jets are at the same Reynolds number. Since the dynamic viscosity $\mu_{j}$ increases with the temperature, Eq. (3) implies that the net thrust is higher at a higher temperature. The temperatures of the jets also lead to different values for the speed of sound, since $a_{j}=\sqrt{\gamma r_{s} T_{j}}$. Therefore, the convective Mach number defined, following Papamoschou \& Roshko [32], as

$$
M_{c}=\frac{M_{j}}{1+a_{\infty} / a_{j}}
$$

also varies. Its value, revealing the importance of compressibility on the development of the mixing layers, is equal to 1 for jetT1Mj2, 1.17 for jetT2Mj2, 1.33 for jetT4Mj2, 0.82 for jetT2Ma2 and 0.67 for jetT4Ma2. Therefore, compressibility effects are expected to strengthen with temperature for $M_{j}=2$, but to weaken for $M_{a}=2$.

The temperature in some of the present jets reach values higher than $1,000 \mathrm{~K}$, which is beyond the temperature of $600 \mathrm{~K}$ below which the ratio of specific heats $\gamma$ can be considered as constant [33]. Despite this, for sake of simplicity, the gas in the jet core and the ambient medium is modelled as an ideal gas with $\gamma=1.4$. This is supported by the observation in reference [34] that taking into account the variations with temperature of the specific heat ratio does not significantly affect the Mach wave radiation in supersonic jets, and by the finding of Buchta \& Freund [35] that the pressure skewness near planar shear layer is only weakly affected by changes in the value of $\gamma$. Likewise, following Joseph et al. [36], it has been verified that the initial Mach numbers of the present jets differed from less than 3\% from the ones computed assuming a temperature dependant specific heat ratio. 


\section{B. Numerical methods}

The simulations are very well-resolved large-eddy simulations (LES) performed by solving the compressible Navier-Stokes equations in cylindrical coordinates $(r, \theta, z)$ using high-order finite differences. In the computations, the spatial derivatives are evaluated using a centered fourth-order eleven-point low-dispersion finite-difference scheme [37], and time integration is carried out using a second-order, six-stage Runge-Kutta algorithm [37]. Near the jet axis, the method proposed by Mohseni \& Colonius [38] is applied to avoid singularity at $r=0$. As a result, the first discretization point is placed at a distance $r=\Delta r / 2$ from the jet axis, where $\Delta r$ is the radial mesh spacing close to the jet axis. The azimuthal derivatives are also evaluated using fewer points than permitted by the grid, in order to reduce the constraint on the time-step arising from the use of cylindrical coordinates near the centerline [39]. More precisely, the effective azimuthal resolution is 16 for the row of points nearest to the jet axis. It progressively increases with the radial distance, and reaches $n_{\theta}=256$ for $r=0.25 r_{0}$. At the end of each time-step, a standard, twelfth-order selective filter is applied to remove grid-to-grid oscillations. Another role of the filter is to relax the subgrid-scale energy near the grid cut-off wavenumber. A shock-capturing scheme [40] based on the application of an adaptive filtering procedure is also applied to damp Gibbs oscillations around shocks. Finally, the radiation conditions of Tam \& Dong [41] are applied at the radial boundary of the computational domain in order to allow the acoustic waves to leave the computational domain without causing significant spurious reflections. At the axial inflow and outflow, periodicity conditions are imposed.

\section{Computational parameters}

The mesh used for the five simulations extends over a distance of $240 r_{0}$ in the axial direction, and out to $13 r_{0}$ in the radial direction. It contains $n_{r} \times n_{\theta} \times n_{z}=382 \times 256 \times 9600$ points, which yields a total number of 940 millions points. The axial spacing $\Delta z$ is constant and equal to $\Delta z=0.025 r_{0}$, yielding a maximum wavenumber $k_{z} r_{0} \simeq 62.8$ for a wave discretized using four points per wavelength. The radial spacing varies with the radial distance. It is such that $\Delta r=\Delta z$ on the jet axis, and reaches a minimum of $\Delta r=0.0063 r_{0}$ at $r=r_{0}$. It then progressively increases up to $r=4 r_{0}$, after which it remains constant and equal to $0.05 r_{0}$. Given their low Reynolds number, the computations can be considered as very well resolved large-eddy simulations. Indeed, it has been verified that the sum of the different terms in the kinetic energy budget is small. This high resolution is motivated by the need to accurately capture the steepened waves, but also all the turbulence structures that may be involved in their generation process. The simulations are carried out until a final time equal to $t_{f}=70 r_{0} / u_{j}$ for jetT1Mj2, to $60 r_{0} / u_{j}$ for jetT2Ma2 and jetT4Ma2, and to $100 r_{0} / u_{j}$ for jetT2Mj2 and jetT4Mj2. For all jets, approximately 10,000 time steps are necessary, which consumed 10,000 CPU hours.

\section{Space-averaged statistics}

Unlike for spatially-developing flows, which are statistically homogeneous in time but not in the axial direction, the signals measured in and near the present temporally-developing jets are homogeneous in the $z$ direction but are 
(a)

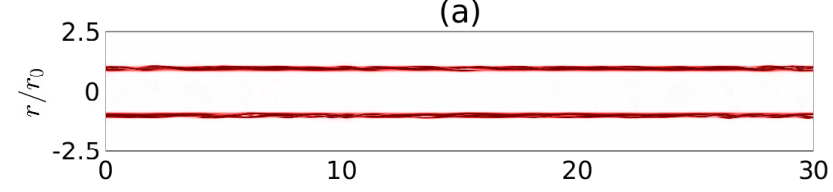

(b)

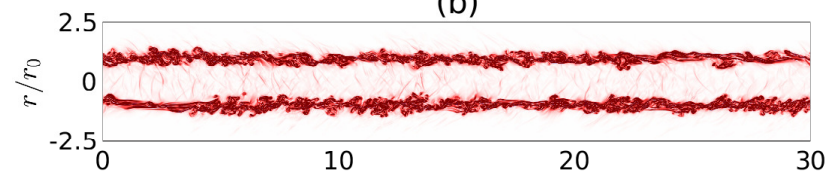

(c)

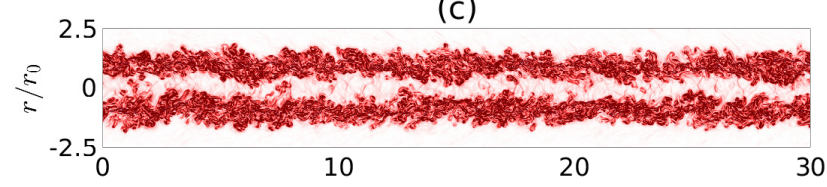

(d)

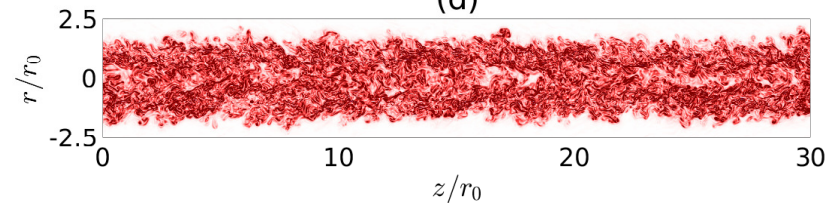

Fig. 3 Vorticity norm for jetT2Mj2 at (a) $t=8 r_{0} / u_{j}$, (b) $16 r_{0} / u_{j}$, (c) $24 r_{0} / u_{j}$, and (d) $32 r_{0} / u_{j}$. The color scale ranges up to $4 u_{j} / r_{0}$, from white to red.

not stationary. For this reason, as in other studies of temporally-developing flows [31, 42], all of the statistical results presented in section III] including the conditional averages in section III.D are computed, for a given time, over the homogeneous directions $z$ and $\theta$. In addition, the convergence of these space-averaged statistics is improved by averaging the results obtained from several runs of the same jet, which are performed by varying the random seed used in the excitation procedure. An illustration of the benefits of this method in the case of a temporal subsonic jet is provided in reference [31]. As reported in table 1 , 4 runs are performed for jetT1Mj2 and 3 runs are performed for the other jets, yielding equivalent domain lengths of $4 \times 240 r_{0}=960 r_{0}$ and $3 \times 240 r_{0}=720 r_{0}$, respectively.

\section{Results}

\section{A. Flow development}

Snapshots of vorticity norm obtained for jetT2Mj2 are given in Fig. 3 at times $t=8 r_{0} / u_{j}, 16 r_{0} / u_{j}, 24 r_{0} / u_{j}$ and $32 r_{0} / u_{j}$. At $t=8 r_{0} / u_{j}$, the jet core is surrounded by thin, laminar mixing layers in which slight perturbations are visible, indicating the development of instability waves. At $t=16 r_{0} / u_{j}$, the mixing layers are thicker than previously and contain more fine-scale turbulence, because of their transition from a laminar to a turbulent state. At subsequent times, the growth of the mixing layers continues until they interact with each other, which is visible at $t=24 r_{0} / u_{j}$ where pockets of vortical flow coming from the shear layers intrude on the jet axis. Finally, at $t=32 r_{0} / u_{j}$, the mixing 
(a)

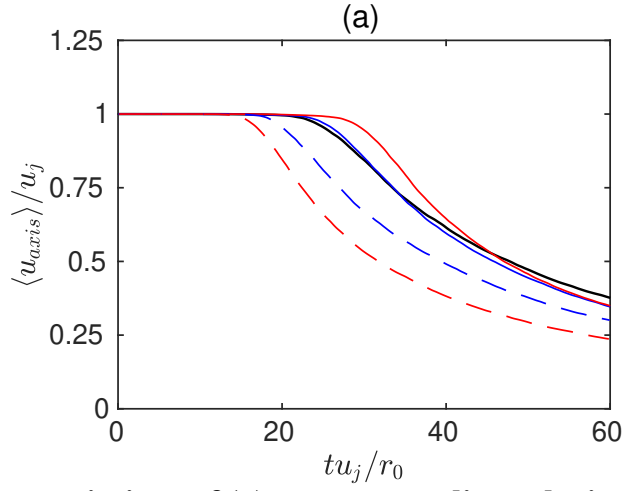

(b)

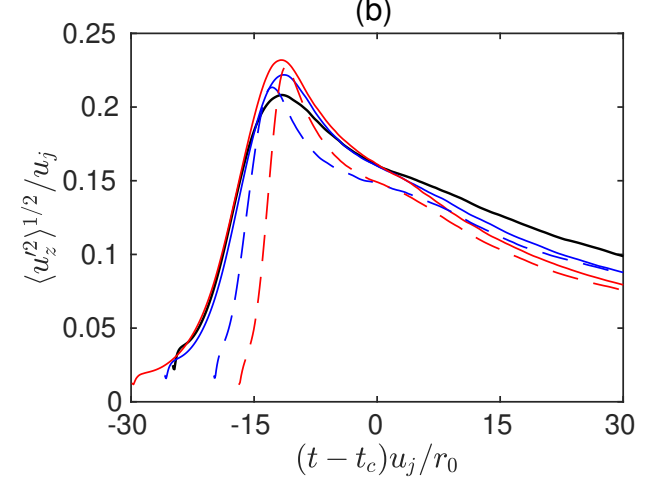

Fig. 4 Time variations of (a) mean centerline velocity and (b) peak turbulence intensities for jetT2Mj2, — jetT4Mj2, - - - jetT2Ma2 and --- jetT4Ma2.

layers join and merge and the inner, potential core of the jet disappears.

The time variations of the mean axial velocity are shown in Fig. 4/a). For all jets, the velocity is constant and equal to its initial value $u_{j}$ at early times and as long as the centerline flow is irrotational. It rapidly decreases afterwards, as low-speed gas is entrained inside the jet core after the merging of the shear layers. The core-closing time $t_{c}$, defined as the time when the centerline mean velocity $\left\langle u_{z, \text { axis }}\right\rangle$ is such that $\left\langle u_{z, a x i s}\right\rangle=0.95 u_{j}$, varies according to the initial conditions. It slightly increases with the temperature for $M_{j}=2$, since $t_{c}=25.3 r_{0} / u_{j}$ for jetT1Mj2, $26.2 r_{0} / u_{j}$ for jetT2Mj2 and $29.8 r_{0} / u_{j}$ for jetT4Mj2, whereas it diminishes at $M_{a}=2$, as $t_{c}=20.2 r_{0} / u_{j}$ for jetT2T14, and 17.0r $0 / u_{j}$ for jetT4Ma2. In order to explore the origin of these different trends, the time variations of the maximum root-mean-square value of axial velocity fluctuations are represented in Fig. $4(\mathrm{~b})$ as a function of $\left(t-t_{c}\right) r_{0} / u_{j}$. In this figure, a peak of $0.2 u_{j}$ is reached for all jets at $t \simeq t_{c}-12 r_{0} / u_{j}$. This peak is caused by pairings between vortices during the transition of the jet from a laminar to a turbulent state [43], which thus occurs in all cases approximately 12 time units before the closure of the potential core. The duration of the initial laminar phase before the pairings strongly depends on the jet considered, as it varies from $5.9 r_{0} / u_{j}$ for jetT4Ma2 to $18.3 r_{0} / u_{j}$ for jetT4Mj2. Consequently, the different core-closing times reported in Fig. 4 a) are mostly due to the different durations of this initial phase which can be explained by compressibility effects. Indeed, as reported in Table 1 , the convective Mach number $M_{c}$ of the jets ranges from 0.67 for jetT4Ma2 to 1.33 for jetT4Mj2. Since the mixing layers growth rate decreases with the convective Mach number [32], it is expected that the initial laminar phase of the jets would last longer for higher values of $M_{c}$, which is observed in the present simulations.

The time variations of the momentum thickness $\delta_{\theta}$ of the shear layers are provided in Fig. 5. Initially, for $t \leq t_{c}-12 r_{0} / u_{j}$, the growth is slow, as the shear layers are in a laminar state. It is faster for $t \geq t_{c}-12 r_{0} / u_{j}$, after their transition to a turbulent state. From that time, and until the closing of the potential core, the momentum thickness of the hot jets increases more rapidly than that of the isothermal one. Since the present jets have different Mach numbers, speeds and densities, the effects of temperature on the jet spreading are not straightforward to interpret. For that reason, 


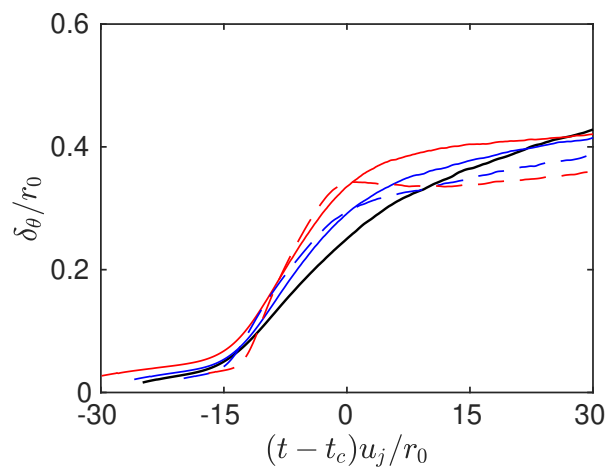

Fig. 5 Time variations of the shear-layer momentum thickness for - jetT1Mj2, — jetT2Mj2, jetT4Mj2, - - - jetT2Ma2 and -- jetT4Ma2.

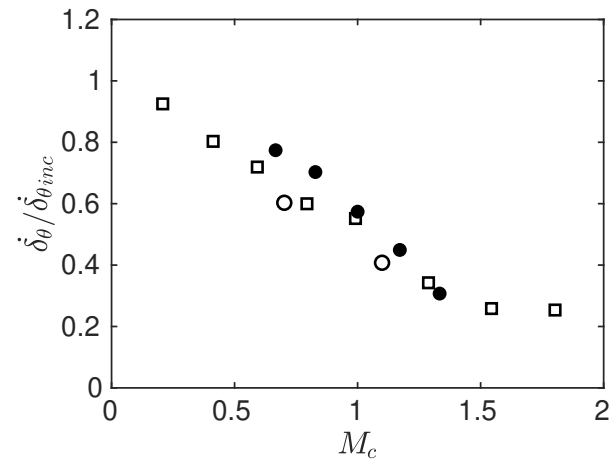

Fig. 6 Momentum-thickness growth rates obtained when $\delta_{\theta}=0.08 r_{0}$ normalized by their incompressible values; • present results, $\square$ Freund et al. [42] and $\circ$ Pantano \& Sarkar [45].

the momentum thickness growth rate $\dot{\delta}_{\theta}=\left(\mathrm{d} \delta_{\theta} / \mathrm{d} t\right) / u_{j}$ is normalized by that obtained for an incompressible mixing layer at the same density ratio, and plotted in Fig. 6 as a function of the convective Mach number $M_{c}$ as in previous studies on compressible mixing layers at different density ratios [32, 44]. The growth rates for the incompressible cases have been determined by simulating three additional temporally-developing round jets at a convective Mach number of 0.15 and at static temperatures of $T_{\infty}, 2 T_{\infty}$ and $4 T_{\infty}$. Since the shear-layer growth rates vary with time, they are displayed in Fig. [6 at a time where $\delta_{\theta}=0.08 r_{0}$ for all jets, following Freund et al. [42]. The normalized growth rate is shown to decrease with the convective Mach number, in agreement with experimental [32] and numerical [42, 45] data, which is a well-known effect of compressibility. The reported values are also in good agreement with those provided in references [42, 45], also plotted for comparison.

The effects of temperature on the mean velocity profiles are characterized by considering the jet half-width $\delta_{05}$, defined as the radial location where $\left\langle u_{z}\right\rangle=0.5 u_{j}$ in Fig. 7 (a). For all jets, it is initially equal to $\delta_{05}=r_{0}$, as expected given the hyperbolic-tangent shape of the profiles at $t=0$. At subsequent times, the half-width of the isothermal jet remains close to $r_{0}$ until $t=t_{c}$, whereas those of the hot jets decrease until they reach the values of $\delta_{05}=0.80 r_{0}$ for jetT2Ma2, $0.68 r_{0}$ for jetT4Ma2, $0.84 r_{0}$ for jetT2Mj2 and $0.74 r_{0}$ for jetT4Mj2 at $t \simeq t_{c}$. Consequently, the half-velocity point is shifted toward the high-speed, low-density side of the mixing layers at a higher jet temperature. This effect is 
(a)

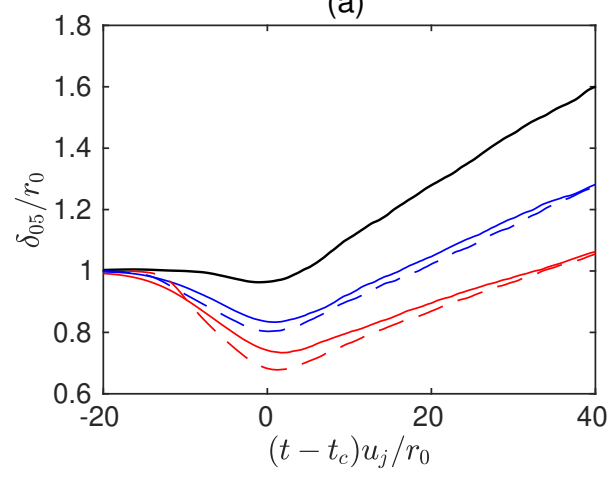

(b)

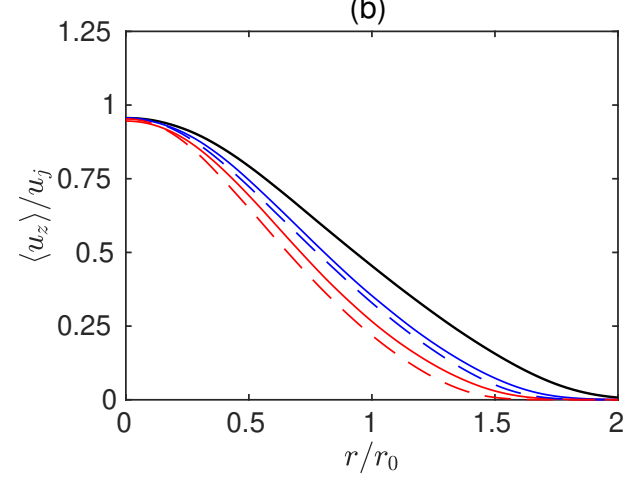

Fig. 7 Representations of (a) jet half-width and (b) mean velocity profile at core-closing time for jetT1Mj2, — jetT2Mj2, — jetT4Mj2, --- jetT2Ma2 and $--{ }_{-}$jetT4Ma2.

illustrated in Fig. 7 (b), where the mean velocity profiles obtained at the core-closing time are plotted. The profiles bend toward the jet axis when the temperature is raised, which has also been observed in studies of plane temporal mixing layers at different density ratios [45], as well as in experimental studies of spatially-developing hot jets at high Reynolds numbers [46].

\section{B. Near pressure field}

The time variations of the pressure levels computed at $r=8 r_{0}$ from the jet axis are plotted in figure 8 (a) as a function of $\left(t-t_{c}\right) a_{\infty} / r_{0}$. For all jets, a peak is reached after the core closing time, at approximately $t=t_{c}+7 r_{0} / a_{\infty}$. The peak of pressure levels is of $1500 \mathrm{~Pa}$ for jetT1Mj2, $970 \mathrm{~Pa}$ for jetT2Ma2 and $650 \mathrm{~Pa}$ for jetT4Ma2. It thus decreases with temperature when the jet speed is constant, in agreement with the experimental results of Tanna et al. [2]. A different trend is however observed for a constant Mach number $M_{j}$. In that case, the peak level is of $1900 \mathrm{~Pa}$ for jetT2Mj2 and $2100 \mathrm{~Pa}$ for jetT4Mj2, and is thus higher at a higher temperature, as observed by Seiner et al. [3] for jets at $M_{j}=2$. This rise of the radiated pressure levels is caused, to a large extent, by the higher velocities of jetT2Mj2 and jetT4Mj2. In order to isolate the effects of temperature, the pressure levels are normalized by $M_{a}^{3 / 2}$ and plotted in Fig. 8(b) assuming, following previous investigators [8,47], that the sound intensity radiated by supersonic jets increases with the third power of the jet speed. The pressure levels obtained for jets at the same static temperature are found to be very close to each others. In addition, the effect of temperature alone is to diminish the intensity of the acoustic waves radiated by the jets. For a constant Mach number $M_{j}$, this effect is however compensated by the rise of the jet speed, leading to higher pressure levels at a higher temperature.

Snapshots of pressure fluctuations and vorticity norm obtained for the five jets are provided in Fig.9 9 at $t=t_{c}+7 r_{0} / a_{\infty}$, very close to the time of peak pressure levels at $r=8 r_{0}$. For all jets, straight, inclined wavefronts, whose associated wavelengths are of the order of the jet diameter are visible near the jets. This observation indicates that the jets radiate Mach waves during their development. For $M_{j}=2$, in Fig. 9 (a,b,d), the inclination angle of the acoustic waves 

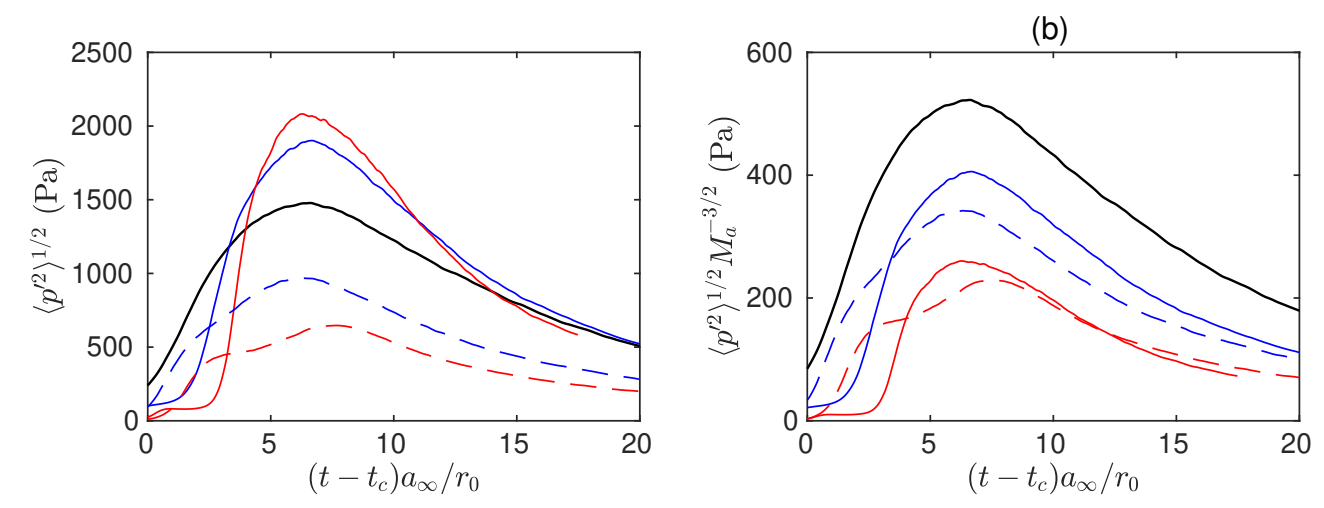

Fig. 8 Time variations of the rms value of pressure fluctuations at $r=8 r_{0}$ for - jetT1Mj2,
jetT2Mj2, - jetT4Mj2, - jetT2Ma2 and $--\ldots$ jetT4Ma2.

significantly increases with temperature, as noticed in the numerical studies of Nonomura et al. [28] and Liu et al. [6], for instance. This increase is mainly explained by the higher velocities of jetT2Mj2 and jetT4Mj2 in comparison with jetT1Mj2. Indeed, no such effect is visible in the snapshots of Fig. 9 a,c,e), for a constant jet speed. In these figures, the inclination angle of the sound waves slightly diminishes with temperature, and the wavefronts radiated by jetT2Ma2 and jetT4Ma2 have a slightly curved aspect. This curved aspect is likely due to the faster decrease of the mean axial velocity, hence convection speed, in that case with respect to the jets at $M_{j}=2$. Finally, it can be noted that steep pressure variations are present at the edges of the wavefronts radiated by the jets. These features are more clearly visible in the sound fields of jetT2Mj2 and jetT4Mj2 than in those of the jets at $M_{a}=2$. This suggests that the hot jets at $M_{j}=2$ radiate more steepened waves than the ones at $M_{a}=2$.

Steepened waves can also be observed in the axial variations of the pressure fluctuations at $r=8 r_{0}$ and $t=t_{c}+7 r_{0} / a_{\infty}$ provided in Fig. 10 for the isothermal jet as well as for jetT4Mj2 and jetT4Ma2. In the three cases, the signals are normalized by their standard deviations in order to facilitate the comparison. For the isothermal jet, in Fig. 10 a), strong pressure peaks emerge as, for instance, at $z=13 r_{0}$ and $z=19 r_{0}$. These peaks consist of a fast compression phase, leading to a pressure peak whose value can exceed three times that of the standard deviation, followed by an expansion phase which is more gradual than the initial compression. The shapes of these shock-like structures are very similar to those depicted by Buchta \& Freund [18] in their simulations of plane temporally-developing mixing layers. They also remind us of those observed in the near and far acoustic fields of spatially-developing supersonic jets [16, 23, 24]. In Fig. 10(b,c), those structures are also present in the sound field of the hot jets, for exemple at $z=13 r_{0}$ for jetT4Ma2, and at $z=8 r_{0}$ and $18 r_{0}$ for jetT4Mj2. For the latter jet, the corresponding pressure peaks reach values as high as five standard deviations, which suggests that the steepened waves constitute stronger deviations from the mean pressure than those generated by the other, slower jets.

The shock-like structures in the pressure signals of Fig. 10 constitute strong deviations which cannot be easily characterized using basic statistics such as sound intensity and power spectrum densities. However, high-order statistics 

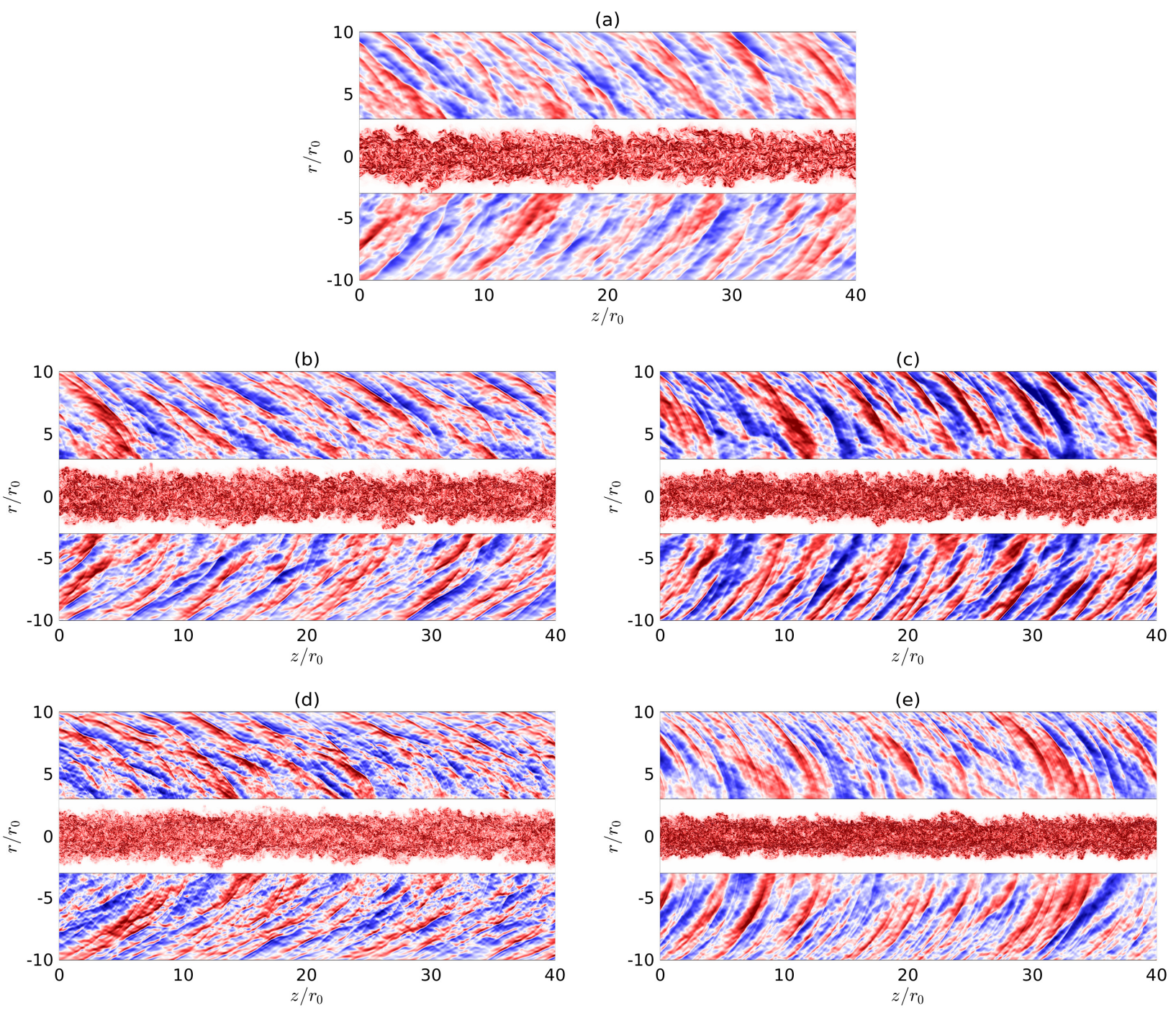

Fig. 9 Pressure fluctuations and vorticity norm at $t=t_{c}+7 r_{0} / a_{\infty}$ for (a) jetT1Mj2, (b) jetT2Mj2, (c) jetT2Ma2, (d) jetT4Mj2 and (e) jetT4Ma2. Color scales between $(a, b, d) \pm 6,000 \mathrm{~Pa}$ and $(\mathrm{c}, \mathrm{e}) \pm 2,000 \mathrm{~Pa}$ for the pressure and up to $3 u_{j} / r_{0}$ for the vorticity.

such as the skewness and kurtosis factors provide useful insights on the properties of such events and have been used in the past to quantify the crackling behavior of high-speed jets [9, 11, 16]. The skewness factor of the pressure fluctuations, defined as

$$
S(p)=\frac{\left\langle p^{\prime 3}\right\rangle}{\left\langle p^{\prime 2}\right\rangle^{3 / 2}}
$$

is a measure of the asymmetry of the pressure fluctuations statistical distribution. Its values obtained at $r=8 r_{0}$ are plotted in Fig. 11 as a function of $\left(t-t_{c}\right) a_{\infty} / r_{0}$. For the jets at $M_{j}=2$, in Fig. 11 (a), the skewness factor is greater than zero for $t_{c}+5 r_{0} / a_{\infty} \leq t \leq t_{c}+10 r_{0} / a_{\infty}$, when the pressure levels are close to their maximum values. During that period, the skewness value is approximately constant and equal to 0.28 for jetT $1 \mathrm{Mj} 2,0.37$ for jetT2Mj 2 and 0.41 for 
(a)

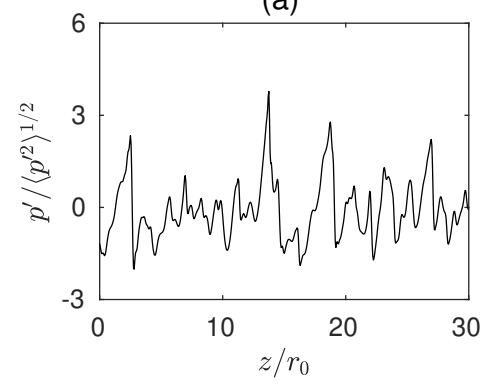

(b)

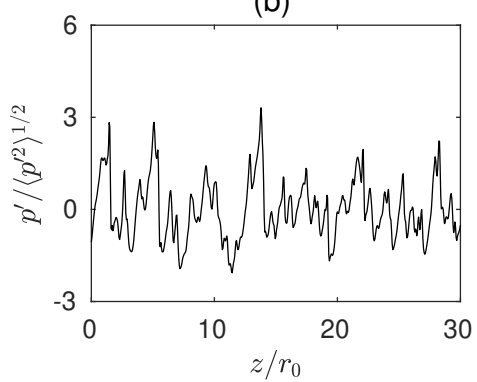

(c)

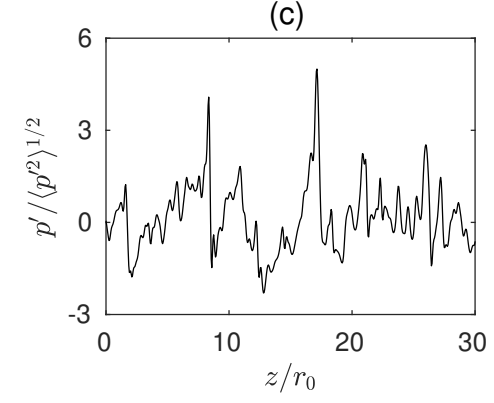

Fig. 10 Pressure fluctuations at $r=8 r_{0}$ and $t=t_{c}+7 r_{0} / a_{\infty}$ normalized by their standard deviation for (a) jetT1Mj2, (b) jetT4Ma2 and (c) jetT4Mj2.

jetT4Mj2. These positive values are caused by the pressure peak associated with the steepened shock-like structures and are higher at a higher temperature, indicating more prominent pressure peaks. No such increase is observed for $M_{a}=2$ in Fig. 11. b). In this case, on the contrary, the skewness factor at time of peak levels, obtained by averaging between $t=t_{c}+5 r_{0} / a_{\infty}$ and $t_{c}+10 r_{0} / a_{\infty}$, is of 0.28 for jetT1Mj2, 0.26 for jetT2Ma2 and 0.18 for jetT4Ma2 and thus slightly decreases with the temperature. It can be noted that the variations of skewness in Fig. 11] occur over time scales of approximately $10 r_{0} / a_{\infty}$ which are longer than the wave turnover time $\lambda / a_{\infty}$, where $\lambda \simeq 2 r_{0}$ is a characteristic length of the pressure waves. Consequently, the skewness does not change significantly as a wave passes through the location of an observer. This allows us to compare the present values with the ones reported in the literature for spatially-developing jets. For instance, Petitjean et al. [24] measured skewness factors ranging from 0.1 to 0.5 in the far acoustic field of heat-simulated jets at acoustic Mach number between 1.2 and 2.5, close to the values reported above for the present temporally-developing jets.

The kurtosis factor of the pressure fluctuations is defined as

$$
K(p)=\frac{\left\langle p^{4}\right\rangle}{\left\langle p^{\prime 2}\right\rangle^{2}}
$$

and is a measure of the probability of occurrence of extreme pressure events, and hence of the signal intermittency. While $K(p)=3$ is obtained for a signal with Gaussian statistics, values higher than 3 indicate that extreme deviations from the mean are more likely to occur. Previous experimental studies of supersonic jets have shown that the kurtosis factor can reach such values in the sound field of highly supersonic jets [9, 16] due to the presence of steepened waves. The time variations of the pressure kurtosis factors measured at $r=8 r_{0}$ are plotted in Fig. 12. For all jets, it is approximately constant between $t_{c}+5 r_{0} / a_{\infty}$ and $t_{c}+12 r_{0} / a_{\infty}$. For $M_{j}=2$, in Fig. [12]a), the kurtosis factor is equal to 3.1 for jetT1Mj2, 3.4 for jetT2Mj2 and 3.5 for jetT4Mj2, and thus increases with temperature, in agreement with the measurements of Mora et al. [9]. However, it does not vary significantly at a constant jet speed, only varying between 3.1 and 3.2 for jetT1Mj2, jetT2Ma2 and jetT4Ma2 in Fig. 12 b). 
(a)

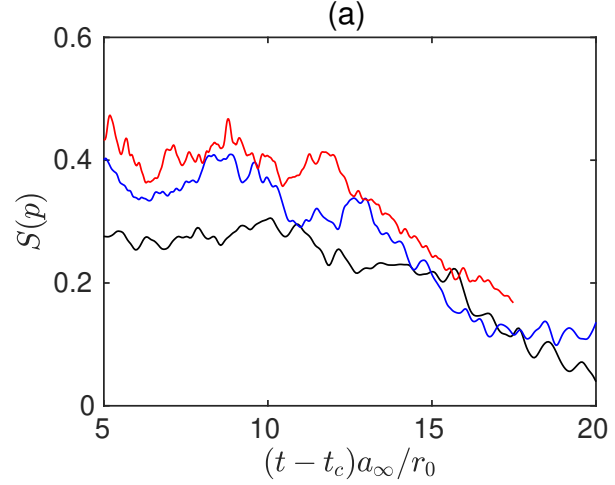

(b)

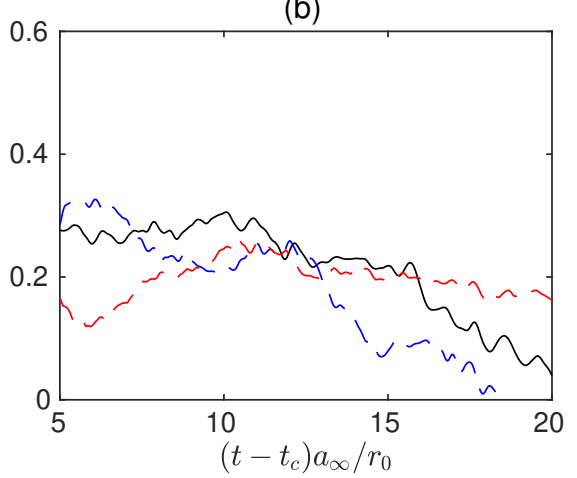

Fig. 11 Skewness factors of the pressure fluctuations at $r=8 r_{0}$ for (a) jetT1Mj2, jetT2Mj2 and jetT4Mj2, and (b)

\section{jetT1Mj}

(a)

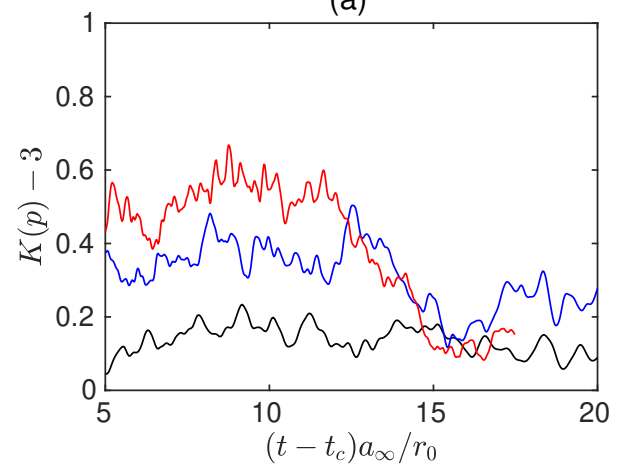

(b)

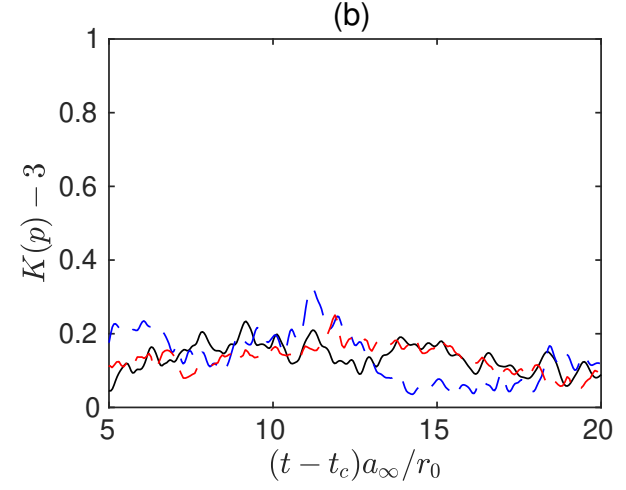

Fig. 12 Kurtosis factors of the pressure fluctuations at $r=8 r_{0}$ for (a) - jetT1Mj2, and $\longrightarrow$ jetT4Mj2, and $(b) \longrightarrow$ jetT1Mj2, -- jetT2Ma2 and --- jetT4Ma2.

Finally, in order to display the effects of the jet speed for a given temperature, the power spectrum densities of the pressure fluctuations at $r=8 r_{0}$ and $t=t_{c}+7 r_{0} / a_{\infty}$ are plotted as a function of the axial wavenumber in Fig. 13 (a) for the jets at $T_{j}=2 T_{\infty}$ and in Fig. 13 b) for $T_{j}=4 T_{\infty}$. They are normalized by $M_{a}^{3}$ assuming, as previously, that the magnitude of the pressure fluctuations increases with the third power of the jet velocity. For the two temperatures considered, the pressure decay in the high-wavenumber range is slower for jetT2Mj2 and jetT4Mj2, resulting in more high-frequency components than for jetT2Ma2 and jetT4Ma2 with lower velocities. This is due to the presence of more high-intensity, steepened waves in the sound field of jetT2Mj2 and jetT4Mj2 than near the other jets at $M_{a}=2$, as observed in previous experimental studies [24, 48]. Finally, it can be noted that the pressure levels differ in the low-wavenumber range, for $k_{z} r_{0} \leq 1$. These differences may be due to the fact that at $r=8 r_{0}$, the pressure field is likely to include hydrodynamic components which are not expected to follow the $M_{a}^{3}$ velocity scaling [49].

\section{Convection speeds}

For the jets at $M_{j}=2$, the rise of the jet speed $u_{j}$ from $2 a_{\infty}$ to $4 a_{\infty}$ mainly explains the higher amplitude of the steepened waves radiated by the jets at a higher temperature. In that case, the convection speed of the large-scale 
(a)

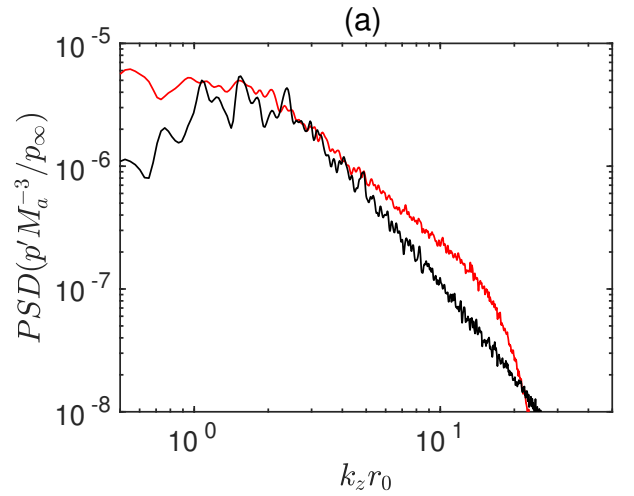

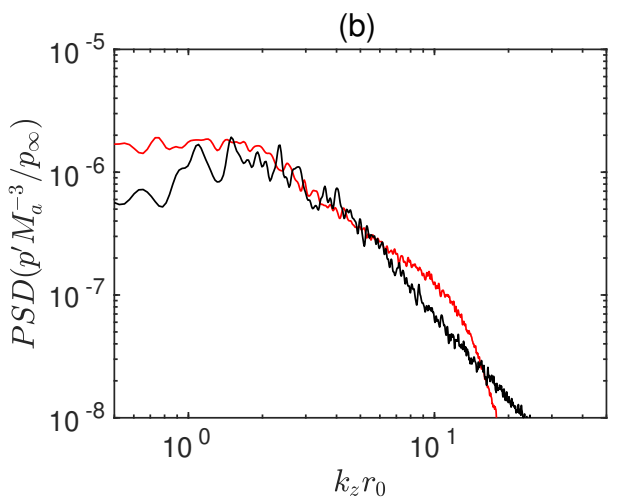

jetT2Mj2,

Fig. 13 Pressure spectra at $r=8 r_{0}$ and $t=t_{c}+7 r_{0} / a_{\infty}$ normalized by $M_{a}^{3}$ for (a) jetT2Ma2, and (b) $\longrightarrow$ jetT4Mj2, jetT4Ma2.

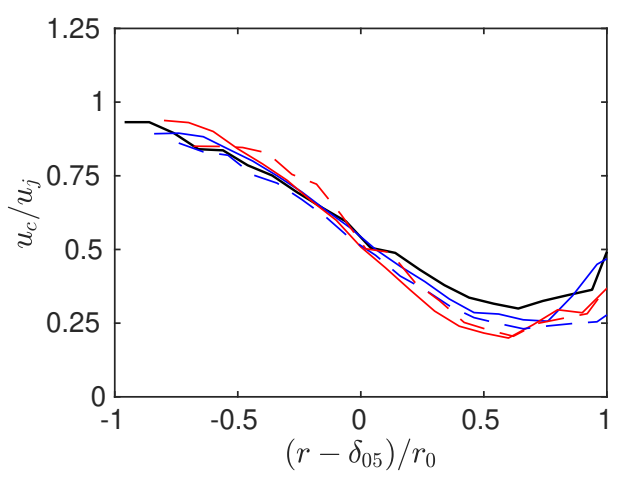

Fig. 14 Radial profiles of the convection speeds at $t=t_{c}$ normalized by $u_{j}$ for jetT2Mj2, — jetT4Mj2, - - jetT2Ma2 and --- jetT4Ma2.

jetT1Mj2,

coherent structures drastically increases, causing the formation of more intense and more skewed Mach waves, as observed in the simulations of Buchta \& Freund [18] for isothermal mixing layers. In order to understand the reason for the slight reduction with temperature of the pressure skewness observed for the jets at $M_{a}=2$, which have identical initial velocities, it seems natural to investigate the convection speeds in their shear-layers. To this end, the latter are first estimated from the space-time correlations of axial velocity fluctuations. The radial variations of the convection speeds obtained at the core-closing time are plotted in Fig. 14(a) as a function of $\left(r-\delta_{05}\right) / r_{0}$, where $\delta_{05}$ is the jet half-width. Despite the differences in temperature, velocity and Mach number, the profiles collapse reasonably well. Since the jet half-width decreases with temperature, as shown in Fig.7 (a), this indicates that the radial profiles of convection velocity are shifted toward the jet axis when temperature is raised. Thus, the convection speed at a given radial location is lower at a lower temperature as in previous studies of spatially-developing hot supersonic jets [6, 50, 51]. This decrease could explain the reduction with temperature of the pressure skewness for the jets at constant speed. However, to confirm this hypothesis, it is necessary to determine the convection speed of the turbulent structures that actually produce the steepened Mach waves. 


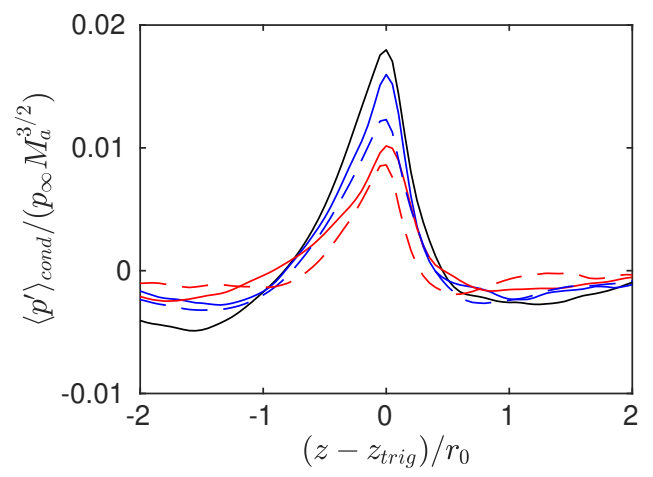

Fig. 15 Conditional pressure waveforms at $r=4.5 r_{0}$ and $t=t_{\text {trig }}=t_{c}+2 r_{0} / a_{\infty}$ jetT2Mj2, — jetT4Mj2, - - jetT2Ma2 and --- jetT4Ma2.

\section{Conditional averages}

Flow features directly linked to noise generation can be obtained by performing correlations between the flow and sound fields, as previously done in studies of temporal [31, 52] and spatial [19, 53] jets. The convection speed can be determined by tracking a specific flow pattern [31, 53] or, in the case of Mach wave radiation, by estimating a mean inclination angle of the wavefronts, yielding an average convection speed using relation (1). This approach will be followed hereafter for the present jets. In practice, the process of steepened waves generation is extracted using conditional averages. To this end, the pressure fluctuations recorded at a distance $r_{\text {trig }}=4.5 r_{0}$ from the jet axis are first binned in intervals of length $3 r_{0}$. The pressure maxima of each subintervals are then determined, and those whose values are lower than twice the standard deviation are discarded. In a second step, the flow and sound field are synchronized according to the axial and azimuthal coordinates $z_{\text {trig }}$ and $\theta_{\text {trig }}$ of the detected maxima. Finally, they are ensemble averaged in order to highlight the flow features that are correlated with the trigger pressure peaks. For all jets, the conditional averaging procedure is applied at time $t_{\text {trig }}=t_{c}+2 r_{0} / a_{\infty}$, close to the peak of pressure levels at $r=r_{\text {trig }}$. In order to save memory storage, it is not applied to the full three-dimensional fields, but to a simplified reconstruction involving only the first five azimuthal Fourier modes. This simplification is motivated by previous observations [22, 54] that the sound waves radiated by highly supersonic jets are strongly correlated in the azimuthal direction.

The conditional pressure signals obtained at $r_{\text {trig }}$ and at time $t_{\text {trig }}$ for the five jets are plotted in Fig. 15 . They are computed by synchronizing approximately 150 events, and are normalized by $p_{\infty} M_{a}^{3}$. The signals obtained for the different jets are very similar, and all peak for $z=z_{\text {trig }}$, as expected given the spatial synchronization. The distinctive features of the sharp pressure waves depicted in the signals of Fig. 10 are recovered. Indeed, a fast compression and a gradual expansion are visible for $z \geq z_{\text {trig }}$ and $z \leq z_{\text {trig }}$, respectively. The pressure peaks are also weaker for the hot jets than for the isothermal jet, following the decrease with temperature of the normalized pressure levels in Fig. 8 (b).

The conditional averages are also evaluated at times $t<t_{\text {trig }}$ by synchronizing the flow and sound fields according to the pressure maxima obtained at $t=t_{\text {trig }}$, which provides a sequential description of the wave generation process. 
(a)
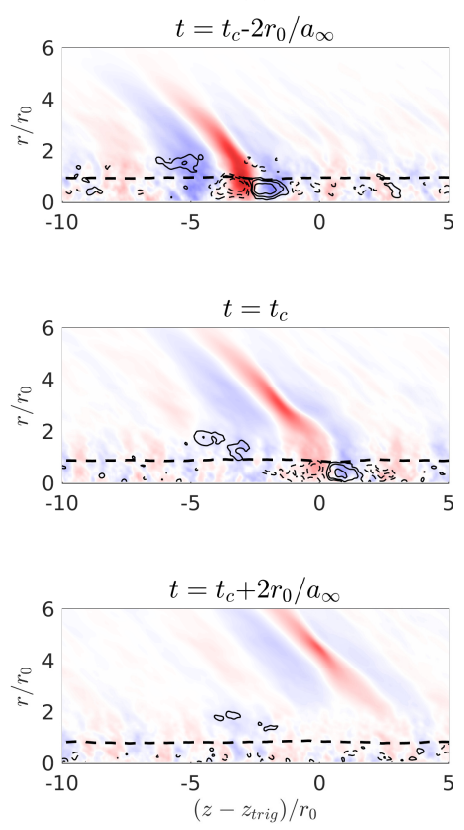

(b)
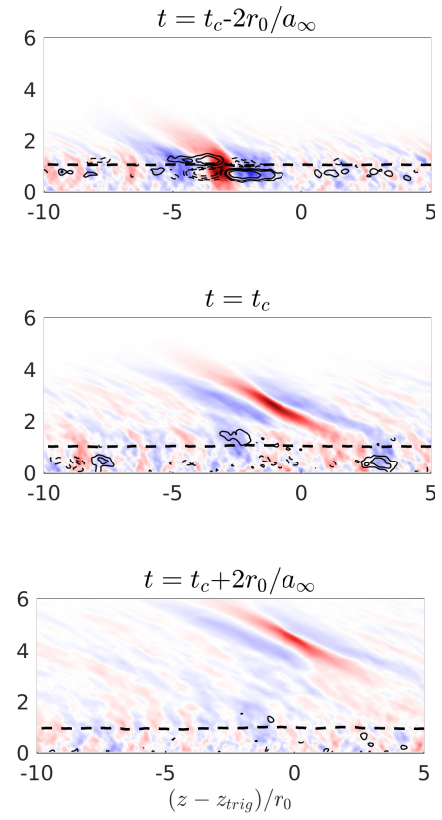

(c)
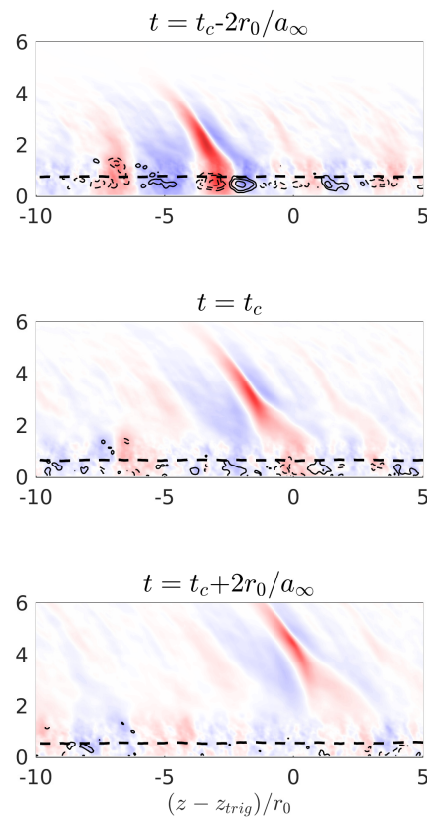

Fig. 16 Conditional pressure and vorticity for (a) jetT1Mj2, (b) jetT4Mj2, and (c) jetT4Ma2; — positive and --- negative vorticity contours, and --- sonic line. Color scales between $(a, b) \pm 12,000$ Pa and (c) \pm 5,000 Pa, from blue to red.

The resulting conditional pressure and vorticity fields are shown in Fig. 16 for jetT1Mj2 as well as for the two hottest jets jetT4Mj2 and jetT4Ma2 at $t_{c}-2 r_{0} / a_{\infty}, t_{c}$ and $t_{\text {trig }}=t_{c}+2 r_{0} / a_{\infty}$. Positive contours of vorticity for the values $0.24 u_{j} / r_{0}, 0.48 u_{j} / r_{0}$ and $0.96 u_{j} / r_{0}$ are represented as black solid lines, and negative contours for the values $-0.96 u_{j} / r_{0}$, $-0.48 u_{j} / r_{0}$ and $-0.24 u_{j} / r_{0}$ are represented as black dashed lines. Some features common to the five jets are discernible. In all cases, an inclined, elongated wavefront emerges from the jet flow at $t_{c}-2 r_{0} / a_{\infty}$. It consists of a band of strong positive levels surrounded by two strips of weaker negative levels. At the lower tip of the wave, an excess of conditional vorticity associated with a pressure deficit is visible inside the jet flow. This zone of positive vorticity has an elliptical shape, and it is located below the sonic line, in the inner core of the jets. Slightly upstream of this vorticity spot, a region of negative vorticity and positive pressure is also apparent. At $t_{c}$, the conditional pressure wave moves outward from the jet flow, and sharp pressure gradients are noticeable at its edge. Inside the jet, the vorticity excess is found downstream from its initial position. Finally, at $t_{c}+2 r_{0} / a_{\infty}$, the pressure wave leaves the flow and propagates downstream, while the vortical structure disappears. These features indicate that the steepened waves observed in the near field constitute a particular case of Mach wave radiation. The vorticity spot can indeed be viewed as the trace of large-scale coherent structures convected by the flow. When their convection speed is higher than the ambient sound speed, they act upon the surrounding medium as a moving wavy-wall, leading to the formation of Mach waves, which propagate in the direction of the angle $\alpha$ given by equation (11). For flow perturbations of moderate amplitude, the formation and the propagation 


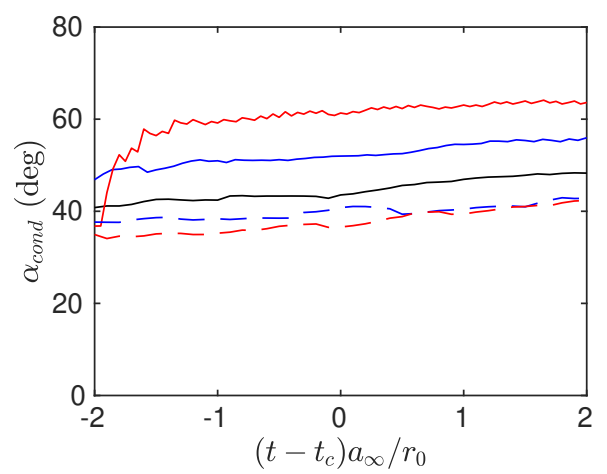

Fig. 17 Time variations of the conditional wavefront orientation angle at the location of minimum dilatation for $\longrightarrow$ jetT1Mj2, — jetT2Mj2, — jetT4Mj2, - - jetT2Ma2 and - - - jetT4Ma2.

of these waves are usually described as linear phenomena, which constitutes the main hypothesis in the classical models of Mach wave radiation [55-57]. However, when nonlinear effects are taken into account, as in the study of Buchta \& Freund [35], who simulated the nonlinear development of a linear instability wave, weak shocks possessing the main features of crackle can be formed in the immediate vicinity of the jet flow. Finally, the present conditional averages are very close to those presented in Appendix, which are obtained by applying the procedure described above to a simulation of a spatially-developing isothermal jet at Mach 2 and at a diameter-based Reynolds number of 12,500. This supports that the mechanisms responsible for the formation of steepened waves in temporal flows do not significantly differ from those in spatially-developing jets.

Despite the similarities of the conditional flow and sound fields, the inclination angle of the waves is largest in Fig. 16 for jetT4Mj2 and lowest for jetT4Ma2. More quantitatively, the inclination angle $\alpha_{\text {cond }}$ of the conditional wavefronts is evaluated by computing the angle of the pressure gradient relatively to the jet direction following

$$
\alpha_{\mathrm{cond}}=\tan ^{-1}\left(\frac{\partial p / \partial r}{\partial p / \partial z}\right) .
$$

The angle $\alpha_{\text {cond }}$ computed for the different jets at the location of minimum dilatation, where the pressure gradient is strongest, is plotted in Fig. 17 as a function of $\left(t-t_{c}\right) a_{\infty} / r_{0}$. For $t \geq t_{c}-r_{0} / a_{\infty}$, the angle $\alpha_{\text {cond }}$ does not change much over time. It increases with temperature for $M_{j}=2$ but decreases for $M_{a}=2$, which is consistent with the trends obtained in the snapshots of Fig.9. A convection speed directly associated with noise generation is then defined from the angle $\alpha_{\text {cond }}$ using relation (11). This convection speed, time averaged between $t_{c}-r_{0} / a_{\infty}$ and $t_{c}+2 r_{0} / a_{\infty}$, ranges from $u_{c}=1.3 a_{\infty}$ for jetT4Ma2 up to $u_{c}=2.1 a_{\infty}$ for jetT4Mj2. As previously, the effects of temperature can be highlighted by considering the ratio between the convection speed and the jet velocity $u_{j}$. This ratio is equal to 0.72 for jetT1Mj2, 0.61 for jetT2Mj2, 0.54 for jetT4Mj2, while it is of 0.66 for jetT2Ma2 and 0.64 for jetT4Ma2. Therefore, the effects of temperature is to diminish the ratio between the convection speed and the jet velocity.

Finally, the influence of the convection speed for the generation of steepened waves is displayed in Fig. 18, where 
(a)

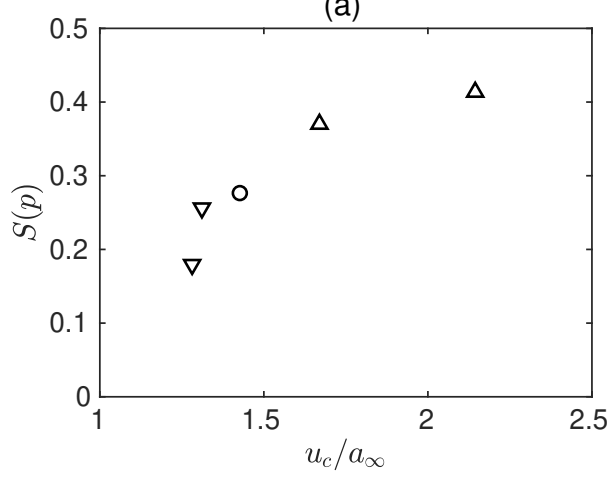

(b)

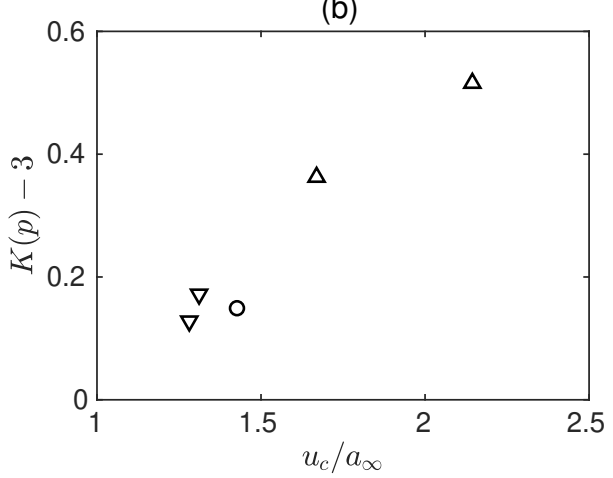

Fig. 18 Time-averaged pressure (a) skewness and (b) kurtosis factors as a function of the convection velocity inferred from the conditional averages for $\circ$ jetT1Mj2 and for the hot jets at $\Delta M_{j}=2$ and $\nabla u_{j}=2 a_{\infty}$.

the pressure skewness and kurtosis factors at $r=8 r_{0}$, time-averaged from $t_{c}-5 r_{0} / a_{\infty}$ to $t_{c}+10 r_{0} / a_{\infty}$, are represented as a function of the convection speed obtained above. In Fig. 18,a), the skewness factor continuously increases with the convection speed, indicating that the acoustic waves radiated by the jets are more skewed as the turbulent structures inside the jet convect more rapidly. In Fig. 18(b), the kurtosis factor obtained for the jets at $M_{a}=2$ is close to 3.1 , but drasticaly increases with the convection speed for $M_{j}=2$, which means that strong deviations are also more likely to occur at a higher convection speed. Therefore, the present results strongly suggest that the steepened aspect of the waves radiated by jets at different temperatures is essentially governed by the convection speed of the turbulent structures at the origin of Mach waves.

\section{Conclusion}

In the present study, the effects of temperature on the formation of steepened acoustic waves near high-speed supersonic jets are investigated by performing numerical simulations of temporal jets at static temperatures equal to 1,2 and 4 times that of the ambient medium. One isothermal and four hot jets are considered, which allows us to investigate separately the effects of a temperature rise at a constant Mach number, as is done in most experimental studies, or at a constant jet speed. When the Mach number is constant, the acoustic waves radiated by the jets are stronger at a higher temperature, and their steepened aspect is more pronounced, which is reflected in the rise of the pressure skewness and kurtosis factors. On the contrary, when the temperature is raised at a constant speed, the pressure levels and skewness factor diminish while the kurtosis is not significantly affected. The two different trends obtained at a constant Mach number or at a constant jet speed are explained by considering the variations with temperature of the convection speed estimated from the autocorrelation functions of the velocity fluctuations. When the temperature is raised, the radial profiles of convection speed are shifted toward the jet axis leading, for a given radial location, to lower values of the ratio between the convection speed and the jet velocity. The importance of the convection speed in the formation of steepened waves is highlighted by performing conditional averages, which shows a direct link between the formation of 
those waves and the supersonic convection of large-scale structures. These averages also allow us to determine, for each jet, a convection speed from the inclination angle of the conditional waves. This convection speed, which is directly related to noise generation, decreases with temperature when normalized by the jet velocity. Among the various effects of increasing temperature on the present flow, which include changes in the Mach number, the density or the thrust of the jets, the decrease of the normalized convection speed appears to be one of the most important temperature effects related to the formation of steepened waves in the jet near field. For a temperature rise at a constant speed, it causes a reduction of the convection velocity, leading to the formation of less steep Mach waves at higher temperature. When the Mach number is constant, this reduction is however compensated by the rise of the jet velocity, so that the convection speed increases with temperature, causing the formation of more intense steepened waves.

\section{Appendix}

In this Appendix, the conditional averaging procedure described in section III.D is applied to the flow and sound fields of a spatially-developing Mach 2 isothermal round jet at a diameter-based Reynolds number of 12,500 in order to verify that the flow features revealed for the present temporally-developing jets are also observed in spatially-developing jets. This jet is computed using the same numerical methods as those presented in section II.B using a grid resolution comparable to that of the present temporal simulations. The jet exhausts at ideally-expanded conditions from a straight-pipe nozzle in which a Blasius laminar boundary layer profile of momentum thickness $\delta_{\theta}=0.018 r_{0}$ is imposed. At each time step, slight velocity fluctuations [58] are added inside the nozzle in order to favor the transition of the shear layers from a laminar to a turbulent state. The amplitude of these perturbation has been tuned such that the turbulence rates at the nozzle exit are of 3\%. Therefore, the shear layers of the jet are initially in a weakly disturbed state, so that the exhaust conditions are very close to those of jetT1Mj2. More details on the simulation are available in reference [19]. A snapshot of the pressure fluctuations and vorticity norm obtained are represented in Fig. 19. Notably, straight, inclined Mach waves are visible near the jet flow and display sharp variations indicating the presence of steepened waves.

The conditional averaging procedure applied to the spatial jet differs from the one applied to the temporal jets since the shock events are now detected in the temporal pressure signals acquired at a particular position, represented as a black cross in Fig. 19 The radial coordinate $r_{\text {trig }}$ of this position is equal to $4.5 r_{0}$, as for the conditional averages computed in section 19 and its axial location $z_{\text {trig }}$ is equal to $17.5 r_{0}$ and corresponds to that of peak pressure fluctuations at $r=r_{t r i g}$. The conditional averages are computed by synchronizing the temporal signals according to the pressure maxima. To this end, the signal acquired at $\left(r_{\text {trig }}, z_{\text {trig }}\right)$ is splitted in intervals of length $3 r_{0} / u_{j}$ and, for each interval, the time $t_{\text {trig }}$ and azimuthal location $\theta_{\text {trig }}$ of the pressure maxima that are higher than twice the standard deviation constitute the reference according to which the signals are synchronized. Then, as in section III.D, the synchronized flow and sound fields are ensemble-averaged at times $t \leq t_{\text {trig }}$ in order to describe the events leading to the formation of one generic, steepened wave. Finally, as for the averages in section III.D, the procedure is applied to a reconstruction of the flow and 


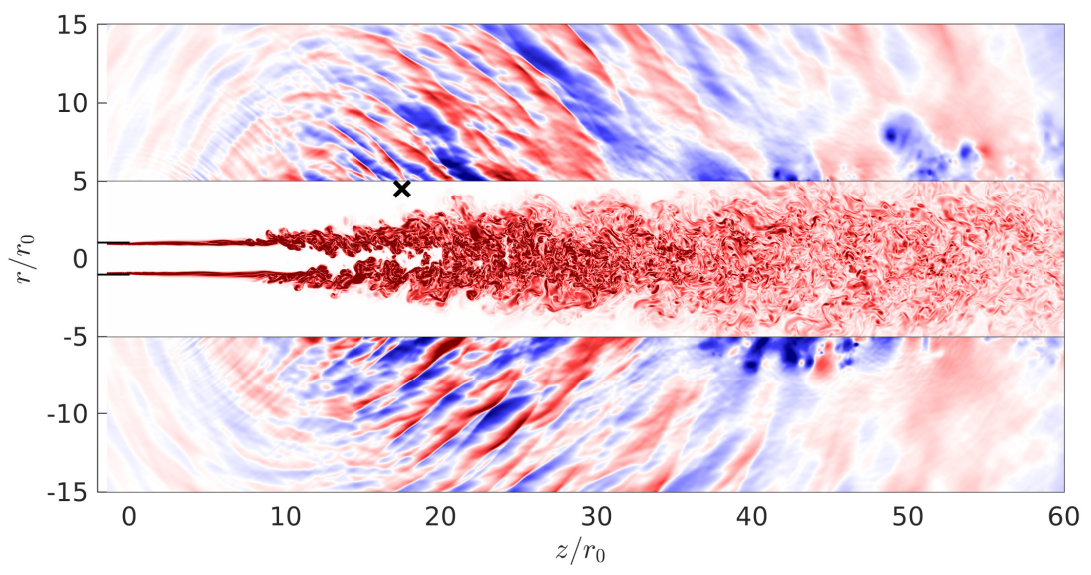

Fig. 19 Snapshots of pressure fluctuations and vorticity norm for a Mach 2 isothermal jet at $R e_{D}=12,500$. The scales range from $-4,000$ to $4,000 \mathrm{~Pa}$ for the pressure and from 0 up to $3 u_{j} / r_{0}$ for the vorticity.

sound field involving the first five azimuthal Fourier modes and are computed using approximately 150 events.

The conditionally-averaged pressure fluctuations are represented in Fig. 20] at $t_{\text {trig }}-6 r_{0} / a_{\infty}, t_{\text {trig }}-3 r_{0} / a_{\infty}$ and $t_{\text {trig }}$, along with the conditional vorticity and the location of the sonic line, defined as $\left\langle u_{z}\right\rangle=u_{j}$. The features revealed in the averaged fields are very similar to those described in Fig. 16. a) for jetT1Mj2. In particular, the pressure waves are connected to a large-scale vorticity excess located below the sonic line and convected downstream by the jet flow. This supports that the mechanisms leading to the formation of steepened waves in temporally-developing jets do not significantly differ from those in spatially-developing ones.

This work was granted access to the HPC resources of FLMSN (Fédération Lyonnaise de Modélisation et Sciences Numériques), partner of EQUIPEX EQUIP@MESO, and of the resources of IDRIS (Institut du Développement et des Ressources en Informatique Scientifique) under the allocation 2018-2a0204 made by GENCI (Grand Equipement National de Calcul Intensif). It was performed within the framework of the Labex CeLyA of Université de Lyon, within the programme 'Investissements d'Avenir' (ANR-10-LABX-0060/ANR-16-IDEX-0005) operated by the French National Research Agency (ANR).

[1] Tanna, H. K., Dean, P. D., and Fisher, M. J., "Influence of temperature on shock-free supersonic jet noise," Journal of Sound and Vibration, Vol. 39, No. 4, 1975, pp. 429-460. doi https://doi.org/10.1016/S0022-460X(75)80026-5

[2] Tanna, H. K., "An experimental study of jet noise. Part 1 : turbulent mixing noise," Journal of Sound and Vibration, Vol. 50, No. 3, 1977, pp. 429-444. doi https://doi.org/10.1016/0022-460X(77)90493-X 

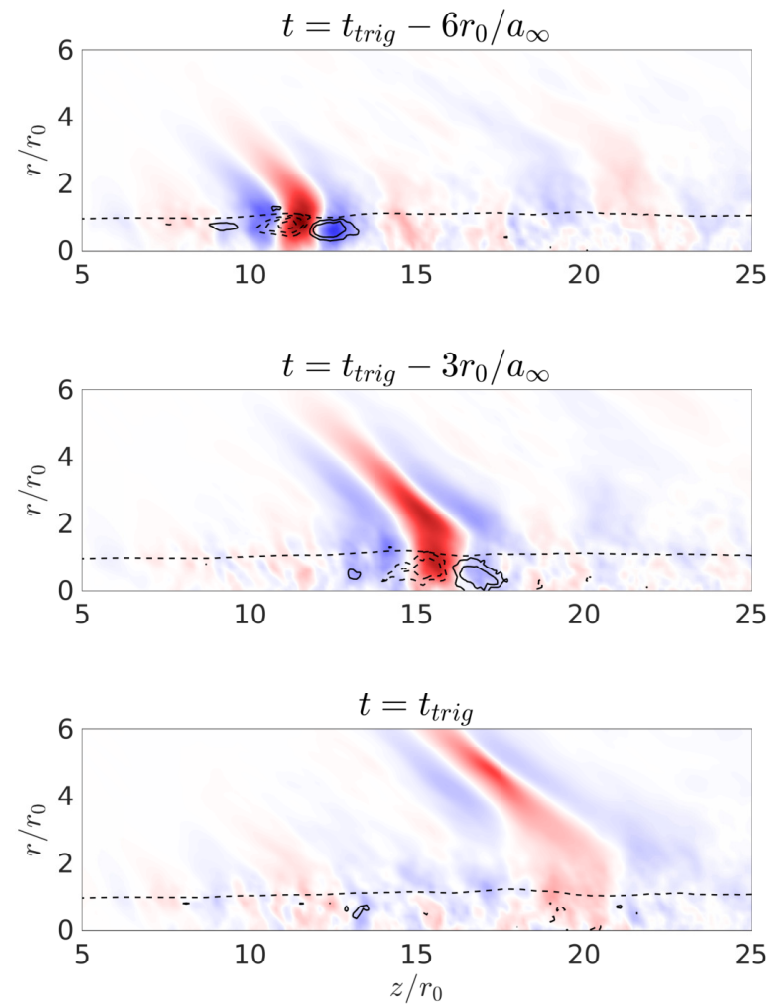

Fig. 20 Conditional pressure and vorticity fields for a Mach 2 spatially developing jet. See Fig. 16 for legend.

[3] Seiner, J., Ponton, M. K., Jansen, B., and Lagen, N., "The effects ot temperature on supersonic jet noise emission,” In 14th DGLR/AIAA aeroacoustics conference, Vol. 1, 1992, pp. 295-307.

[4] Krothapalli, A., Venkatakrishnan, L., and Lourenco, L., "Crackle: A dominant component of supersonic jet mixing noise,” AIAA paper 2000-2024, 2000. doi https://doi.org/10.2514/6.2000-2024

[5] Viswanathan, K., “Aeroacoustics of hot jets,” Journal of Fluid Mechanics, Vol. 516, 2002, pp. 39-82. doi https://doi.org/10.1017/S0022112004000151

[6] Liu, J., Corrigan, A., Kailasanath, K., and Gutmark, E., "Effects of temperature on noise generation in supersonic jets," AIAA paper 2016-2937, 2016. doi:https://doi.org/10.2514/6.2016-2937

[7] Kearney-Fischer, M., Kim, J. H., and Samimy, M., “A study of Mach wave radiation using active control,” Journal of Fluid Mechanics, Vol. 681, 2011, pp. 261-292. doi/https://doi.org/10.1017/jfm.2011.196

[8] Greska, B., Krothapalli, A., Horne, W. C., and Burnside, N., "A near-field study of high temperature supersonic jets," AIAA paper 2008-3026, 2008. doi https://doi.org/10.2514/6.2008-3026

[9] Mora, P., Heeb, N., Kastner, J., Gutmark, E. J., and Kailasanath, K., "Impact of heat on the pressure skewness and kurtosis in supersonic jets," AIAA Journal, Vol. 52, No. 4, 2014. doi https://doi.org/10.2514/1.J052612 
[10] Panda, J., "Experimental investigation of turbulent density fluctuations and noise generation from heated jets," Journal of Fluid Mechanics, Vol. 591, 2007, pp. 73-96. doi:https://doi.org/10.1017/S0022112007007173.

[11] Ffowcs Williams, J. E., Simson, J., and Virchis, V. J., "'Crackle': an annoying component of jet noise," Journal of Fluid Mechanics, Vol. 71, 1975, pp. 251-271. doi https://doi.org/10.1017/S0022112075002558

[12] Gee, K. L., Sparrow, V. W., James, M. M., Downing, J. M., Hobbs, C. M., Gabrielson, T. B., and Atchley, A. A., “The role of nonlinear effects in the propagation of noise from high-power jet aircraft," Journal of the Acoustical Society of America, Vol. 123, No. 6, 2008, pp. 4082-4093. doi https://doi.org/10.1121/1.2903871.

[13] Petitjean, B., and McLaughlin, D. K., "Experiments on the nonlinear propagation of noise from supersonic jets," AIAA paper 2003-3127, 2003. doi https://doi.org/10.2514/6.2003-3127

[14] de Cacqueray, N., and Bogey, C., "Noise of an overexpanded Mach 3.3 jet: non-linear propagation effects and correlations with flow," International Journal of Aeroacoustics, Vol. 13, No. 7-8, 2014, pp. 607-632. doi https://doi.org/10.1260/1475472X.13.7-8.607

[15] Papamoschou, D., "Evidence of shocklets in a counterflow supersonic shear layer," Physics of Fluids, Vol. 7, No. 2, 1995, pp. 233-235. doi https://doi.org/10.1063/1.868621

[16] Fiévet, R., Tinney, C. E., Baars, W. J., and Hamilton, M. F., "Coalescence in the sound field of a laboratory-scale supersonic jet," AIAA Journal, Vol. 54, No. 1, 2016, pp. 254-265. doi https://doi.org/10.2514/1.J054252.

[17] Nichols, J. W., Lele, S. K., Ham, F. E., Martens, S., and Spyropoulos, J. T., "Crackle noise in heated supersonic jets," Journal of Engineering for Gas Turbines and Power, Vol. 135, No. 5, 2013. doi http://dx.doi.org/10.1115/1.4007867

[18] Buchta, D. A., and Freund, J. B., "The near-field pressure radiated by planar high-speed free-shear-flow turbulence," Journal of Fluid Mechanics, Vol. 832, 2017, p. 383-408. doi:10.1017/jfm.2017.671

[19] Pineau, P., and Bogey, C., "Study of the generation of shock waves by high-speed jets using conditional averaging," AIAA paper 2018-3305, 2018. doi https://doi.org/10.2514/6.2018-3305

[20] Baars, W., Tinney, C. E., and Hamilton, M. F., "On cumulative nonlinear acoustic waveform distorsions from high-speed jets," Journal of Fluid Mechanics, Vol. 749, 2014, pp. 331-366. doi https://doi.org/10.1017/jfm.2014.228

[21] Murray, N. E., and Lyons, G. W., “On the convection velocity of source events related to supersonic jet crackle,” Journal of Fluid Mechanics, Vol. 793, 2016, pp. 477-503. doi https://doi.org/10.1017/jfm.2016.127

[22] Pineau, P., and Bogey, C., "Steepened Mach waves near supersonic jets: study of azimuthal structure and generation process using conditional averages" Journal of Fluid Mechanics (accepted), 2019.

[23] Nichols, J. W., Lele, S. K., and Spyropoulos, J. T., “The source of crackle noise in heated supersonic jets,” AIAA paper 2013-2197, 2013. doi https://doi.org/10.2514/6.2013-2197 
[24] Petitjean, B., Viswanathan, K., and McLaughlin, D., "Acoustic pressure waveforms measured in high speed jet noise experiencing nonlinear propagation,” International Journal of Aeroacoustics, Vol. 5, No. 2, 2006 , pp. $193-215$. doi https://doi.org/10.1260/2F147547206777629835

[25] Bogey, C. and Sabatini, R., "Effects of nozzle-exit boundary-layer profile on the initial shear-layer instability, flow field and noise of subsonic jets," Journal of Fluid Mechanics, Vol. 876, 2019, pp. 288-325. doi https://doi.org/10.1017/jfm.2019.546

[26] Brès, G., Jordan, P., Jaunet, V., Le Rallic, M., Cavalieri, A. V. G., Towne, A., Lele, S. K., Colonius, T. and Schmidth, O. "Importance of the nozzle-exit boundary-layer state in subsonic turbulent jets," Journal of Fluid Mechanics, Vol. 851, 2018, pp. 83-124. doi https://doi.org/10.1017/jfm.2018.476

[27] Bogey, C., Marsden, O. and Bailly, C., "Influence of initial turbulence level on the flow and sound fields of a subsonic jet at a diameter-based Reynolds number of 105," Journal of Fluid Mechanics, Vol. 701, 2012, pp. 352-385. doi https://doi.org/10.1017/jfm.2012.162.

[28] Nonomura, T., Nakano, H., Ozawa, Y., Terakado, D., Yamamoto, M., Fujii, K., and Oyama, A., "Large eddy simulation of acoustic waves generated from a hot supersonic jet," Shock waves, 2019, pp. 1-22. doi https://doi.org/10.1007/s00193-019-00895-2.

[29] Bogey, C. and Pineau, P., "Potential-core closing of temporally-developing round jets: Mach number scaling and conditional averaging of flow and sound fields," Physical Review of Fluids, submitted in revised form, 2019.

[30] Zaman, K. B. M. Q., "Effect of initial condition on subsonic jet noise,” AIAA Journal, Vol. 23, No. 9, 1985, pp. $1370-1373$. doi https://doi.org/10.2514/3.9094.

[31] Bogey, C., "On noise generation in low Reynolds number temporal round jets at a Mach number of 0.9," Journal of Fluid Mechanics, Vol. 859, 2019, pp. 1022-1056. doi https://doi.org/10.1017/jfm.2018.864

[32] Papamoschou, D., and Roshko, A., "The compressible turbulent shear layer : an experimental study," Journal of Fluid Mechanics, Vol. 197, 1988, pp. 453-477. doi https://doi.org/10.1017/S0022112088003325

[33] Anderson, J. D., “Modern Compressible Flows : With Historical Perspective”, McGraw-Hill, 2nd edition, 1990, isbn 0-07-100665-6.

[34] Liu, J., Corrigan, A., Kailasanath, K., and Taylor, B., "Impact of the specific hear ratio on noise generation in a high-temperature supersonic jet," AIAA paper 2016-2125, 2016. doi:https://doi.org/10.2514/6.2016-2125

[35] Buchta, D. A., and Freund, J. B., "Intense sound radiation by high-speed flow: Turbulence structure, gas properties, and near-field gas dynamics," Physical Review Fluids, Vol. 4(4), 2019, p.044605. doi 10.1103/PhysRevFluids.4.044605

[36] Joseph, J. G., Tinney, C. E. and Murray, N., "Ideal gas effects in aeroacoustics," AIAA paper 2016-0688, 2016. doi https://doi.org/10.2514/6.2017-0688

[37] Bogey, C., and Bailly, C., "A family of low dispersive and low dissipative explicit schemes for noise computation," Journal of Computational Physics, Vol. 194, No. 1, 2004, pp. 194-214. doi https://doi.org/10.1016/j.jcp.2003.09.003 
[38] Mohseni, K., and Colonius, T., "Numerical treatment of polar coordinate singularities," Journal of Computational Physics, Vol. 157, No. 10, 2002, pp. 3593-3600. doi:https://doi.org/10.1006/jcph.1999.6382

[39] Bogey, C., Marsden, O., and Bailly, C., "Finite differences for coarse azimuthal discretization and for reduction of effective resolution near origin of cylindrical flow equations," Journal of Computational Physics, Vol. 230, 2011, pp. 1134-1146. doi https://doi.org/10.1016/j.jcp.2010.10.031

[40] Bogey, C., de Cacqueray, N., and Bailly, C., "A shock-capturing methodology based on adaptative spatial filtering for high-order non-linear computations," Journal of Computational Physics, Vol. 228, 2009, pp. 1447-1465. doi https://doi.org/10.1016/j.jcp.2008.10.042

[41] Tam, C. K. W., and Dong, Z., "Radiation and outflow boundary conditions for direct computation of acoustic and flow disturbances in a nonuniform mean flow," Journal of Computational Acoustics, Vol. 4, No. 2, 1996, pp. 175-201. doi https://doi.org/10.1142/S0218396X96000040

[42] Freund, J. B., Lele, S. K., and Moin, P., "Compressibility effects in a turbulent annular mixing layer. Part 1. Turbulence and growth rate," Journal of Fluid Mechanics, Vol. 421, 2000, pp. 229-267. doi/https://doi.org/10.1017/S0022112000001622.

[43] Bogey, C., and Bailly, C., "Influence of nozzle-exit boundary-layer conditions on the flow and acoustic fields of initially laminar jets," Journal of Fluid Mechanics, Vol. 663, 2010, pp. 507-538. doi https://doi.org/10.1017/S0022112010003605

[44] Lu, G., and Lele, S. K., "On the density ratio effect on the growth rate of a compressible mixing layer," Physics of Fluids, Vol. 6, 1994, pp. 1073-1075. doi https://doi.org/10.1063/1.868340.

[45] Pantano, C., and Sarkar, S., "A study of compressibility effects in the high-speed turbulent shear layer using direct simulation," Journal of Fluid Mechanics, Vol. 451, 2002, pp. 29-371. doi:https://doi.org/10.1017/S0022112001006978

[46] Lau, J. C., "Effects of exit Mach number and temperature on mean-flow and turbulence characteristics in round jets," Journal of Fluid Mechanics, Vol. 105, 1981, pp. 193-218. doi https://doi.org/10.1017/S0022112081003170

[47] Ffowcs Williams, J. E., “The noise from turbulence convected at high speed,” Philosophical Transactions of the Royal Society of London, Vol. 255, No. 1061, 1963, pp. 469-503. doi https://doi.org/10.1098/rsta.1963.0010

[48] Schlinker, R., Liljenberg, S., Polak, D., Post, K., and Chipman, C., "Supersonic jet noise source characteristics and propagation: engine and model scale," AIAA paper 2007-3623, 2007. doi:https://doi.org/10.2514/6.2007-3623

[49] Arndt, R. E. A., Long, D. F., and Glauser, M. N., "The Proper Orthogonal Decomposition of pressure fluctuations surrounding a turbulent jet," Journal of Fluid Mechanics, Vol. 340, 1997, pp. 1-33. doi https://doi.org/10.1017/S0022112097005089

[50] Ecker, T., Lowe, K. T., and Ng, W. F., "Eddy convection in developing heated supersonic jets," AIAA Journal, Vol. 53, No. 11, 2015, pp. 3305-3315. doi https://doi.org/10.2514/1.J053946

[51] Gojon, R., Baier, F., Gutmark, E., and Mihaescu, M., "Temperature effects on the aerodynamic and acoustic fields of a rectangular supersonic jet," AIAA paper 2017-0002, 2017. 
[52] Pineau, P., and Bogey, C., "Numerical study of the sound fields of temporally-developing supersonic round jets," AIAA paper 2017-3209, 2017. doi https://doi.org/10.2514/6.2017-3209

[53] Bogey, C., "Two-dimensional features of correlations in the flow and near pressure fields of Mach number 0.9 jets," AIAA paper 2019-0806, 2019, pp. 1-14. doi https://doi.org/10.2514/6.2019-0806.

[54] de Cacqueray, N., Bogey, C., and Bailly, C., "Investigation of a High-Mach-Number Overexpanded Jet Using Large-Eddy Simulation,” AIAA Journal, Vol. 49, No. 10, 2011, pp. 2171-2182. doi https://doi.org/10.2514/1.J050952.

[55] Sinha, A., Rodriguez, D., Brès, G., and Colonius, T., "Wavepacket models for supersonic jet noise," Journal of Fluid Mechanics, Vol. 742, 2014, pp. 71-95. doi https://doi.org/10.1017/jfm.2013.660

[56] Tam, C. K. W., and Burton, D. E., "Sound generated by instability waves of supersonic flows. Part 2. Axisymmetric jets," Journal of Fluid Mechanics, Vol. 138, 1984, p. 273-295. doi:https://doi.org/10.1017/S0022112084000124

[57] Tam, C. K. W., and Chen, P., “Turbulent mixing noise from supersonic jets,” AIAA Journal, Vol. 32, No. 9, 1994, pp. $1774-1780$. doi https://doi.org/10.2514/3.12173

[58] Bogey, C. and Marsden, O. and Bailly, C., "Large-eddy simulation of the flow and acoustic fields of a Reynolds number $10^{5}$ subsonic jet with tripped exit boundary layers," Physics of Fluids, Vol. 23, 035104, 2011, pp. 1-20. doi https://doi.org/10.1063/1.3555634 